\title{
THE IHARA ZETA FUNCTION FOR INFINITE GRAPHS
}

\author{
DANIEL LENZ, FELIX POGORZELSKI, AND MARCEL SCHMIDT
}

\begin{abstract}
We put forward the concept of measure graphs. These are (possibly uncountable) graphs equipped with an action of a groupoid and a measure invariant under this action. Examples include finite graphs, periodic graphs, graphings and percolation graphs. Making use of Connes' non-commutative integration theory we construct a Zeta function and present a determinant formula for it. We further introduce a notion of weak convergence of measure graphs and show that our construction is compatible with it. The approximation of the Ihara Zeta function via the normalized version on finite graphs in the sense of Benjamini-Schramm follows as a special case. Our framework not only unifies corresponding earlier results occurring in the literature. It likewise provides extensions to rich new classes of objects such as percolation graphs.
\end{abstract}

\section{Contents}

Introduction

List of essential pieces of notation

1. The framework of measure graphs

1.1. Graphs

1.2. Graphs over groupoids and invariant measures

1.3. Measure graphs

1.4. Some examples of measure graphs

2. The Ihara Zeta function of a measure graph

2.1. The definition and basic properties

2.2. Examples

3. A determinant formula for the Zeta function

3.1. The von Neumann algebra

3.2. The determinant formula

4. Zeta function and integrated density of states on essentially regular graphs

5. Convergence of measure graphs and of Zeta functions

5.1. Weakly convergent graph sequences

5.2. Percolation graphs

6. Actions of sofic groups on graphs

References

\section{INTRODUCTION}

The theory of Zeta functions of finite graphs is a well established topic connecting various branches of mathematics, see e.g. the monograph by Terras [35. Here, the Zeta function

Date: January 10, 2018. 
comes about as function storing information on the number of loops in the graph. More specifically, it is essentially given as exponential of a power series whose $n$ th-coefficient is determined by the number of loops of length $n$. In contrast, Zeta functions on infinite graphs are much less understood. In fact, for general infinite graphs it is not even clear how to define a Zeta function in the first place as - due to the infiniteness of the graph - there are infinitely many loops of each length.

Recent years have seen quite some interest in Zeta functions on infinite graphs. Indeed, for certain periodic graphs an ad-hoc definition of the Zeta function has been given by Clair / Mokhtari-Sharghi in [5] and for certain specific examples it has been investigated how to define a Zeta function via suitable approximations by Grigorchuk / Zuk [16], Clair / MokhtariSharghi [6] and Guido / Isola / Lapidus [19, 20]. The authors of [19] note as a main motivation for their study that there are only very few infinite graphs for which a Zeta function is defined. Also, for the two dimensional integer lattice a Zeta function has been defined and computed by Clair in [7] and for general regular graphs with a transitive group action a Zeta function has been defined and studied in its connection to heat kernels in Chinta / Jorgenson / Karlsson 4]. A recent approach for a class of infinite weighted graphs can be found in [10].

Roughly speaking these works offer two different solutions to deal with the mentioned problem of infiniteness of number of loops of a given size: One solution is to suitably approximate the infinite graph by finite graphs and show convergence of the Zeta functions of the finite graphs [16, 6, 19, 20]. The other solution amounts to only counting the loops at finitely many special vertices [7, 4] as is very natural in the presence of symmetries in the graph.

While these offer very convincing solutions in specific cases, there is so far no general procedure on how to associate a Zeta function to a graph or how to approximate it and there is no closed formula for a Zeta function on a general graph. This is the starting point for our paper. Our main aims are the following:

- To associate an Ihara type Zeta function to a large class of graphs (called measure graphs below) containing finite, periodic, percolation graphs and graphings as subclasses.

- To provide a closed formula for this Zeta function via determinants on von Neumann algebras.

- To study the continuous dependence of this Zeta function on the underlying measure graph.

The corresponding results are all new in the general context provided here. On the one hand, they give a systematic and unified foundation for the works mentioned above. On the other hand, they also extend the framework studied in the literature in various ways. For instance, graphs obtained by vertex percolation on Cayley graphs fall into the studied class. Consequently, one obtains the - to the knowledge of the authors - first approach to define the Ihara Zeta function for percolation graphs.

As part of our investigation

- we put forward a notion of weak convergence of measure graphs.

Weak convergence of measure graphs may be of independent interest. It contains the concept of Benjamini-Schramm convergence for finite graphs as a special case. Moreover, it also allows for convergence of infinite graphs. For example, we show that percolation graphs with weakly convergent probability laws are weakly convergent as measure graphs. Another advantage of weak convergence of measure graphs concerns the description of the limiting object. More precisely, when dealing with sofic Cayley graphs or periodic graphs we are able to directly 
obtain the original graph as the limit object from a weakly convergent sequence of finite measure graphs.

In our context, weak convergence of measure graphs allows us to settle the issue of the continuous dependence of the Zeta function on the underlying measure graph. More specifically, we establish that weak convergence of measure graphs implies convergence of the corresponding Zeta functions. From this continuity result we obtain the previously known approximation results for Zeta functions via finite graphs as a special case. Moreover, as a complete novelty, we obtain from this continuity result the convergence of the Zeta functions associated with a weakly convergent sequence of (infinite) percolation graphs.

To achieve the mentioned aims and results we introduce the concept of a measure graph. Indeed, setting up the framework centered around measure graphs can be seen as the main task in our approach. The idea behind it is simple: Measure graphs provide a measure on the graph and this measure satisfies an invariance property reflecting the symmetries of the graph. This then allows one to calculate an 'averaged number of loops of a given size' by counting in each vertex the loops of this size and then average this function via the given measure. In this way, loops at all vertices are taken into account and at the same time one ends up with a finite number. On the technical level quite some care is required, in particular, in order to implement the invariance of the measure. To do so, we rely on Connes' non-commutative integration theory $[8]$.

Our set-up may be of interest for other questions as well. For example it may be useful for dealing with random Schrödinger operators on graphs.

The present paper is organized as follows. We present our framework in Section 11. In particular, there we introduce the concept of measure graph $(G, M)$ consisting of a measurable graph $G$ together with a measure $M$ on its vertex set. In order to formulate the invariance property of the measure we will need the action of a groupoid $\mathcal{G}$ on $G$. The assumptions required for this action lead us to the concept of a graph over a groupoid.

We then use these ingredients to introduce the concept of the Ihara Zeta function of a measure graph $(G, M)$ in Section 2. The Zeta function is put forward as the exponential of a power series. The coefficients of the power series are determined via Connes' non-commutative integration theory. In this way we effectively obtain these coefficients as integrals over the space of vertices.

Non-commutative integration theory also allows us to introduce von Neumann algebras associated to measure graphs. This is discussed in Section 3 and may, again, be of independent interest in further studies as well. We make use of these tools to prove that the Zeta function can be calculated via a determinant of an operator on the vertices. Specifically, with notation introduced below, Theorem 3.11 gives for each measure graph $(G, M)$ the following.

Determinant formula. $Z_{(G, M)}(u)^{-1}=\left(1-u^{2}\right)^{-\chi_{(G, M)}} \operatorname{det}_{\tau}\left(I-u A_{G}+u^{2} Q_{G}\right)$.

Of course, the use of determinants of non-positive operators requires some care. Here, we essentially use the determinant provided in [19, 20]. For positive invertible operators, this notion coincides with the famous Fuglede-Kadison determinant [15], see also [26]. For possibly non-invertible operators, one has to deal with singularities. Results for such elements in a von Neumann algebra associated with some countable, amenable group have recently been proven by Li / Thom in [24].

In Section 4 we have a look at the case that the vertex degree is constant. In this case the determinant formula can be considerably simplified. It can then be expressed via the so-called integrated density of states. This is the content of Theorem 4.3. The formula proven in the 
theorem has been used in [16 to define a Zeta function. It also has recently been obtained in [4] via an analysis of Bessel functions and heat kernels.

Section 5 is concerned with the convergence of measure graph sequences and the convergence of the corresponding sequence of Ihara Zeta functions. Our notion goes beyond weak convergence of finite graphs in the sense of Benjamini-Schramm. Concerning the convergence of the underlying Ihara Zeta functions, we obtain in Theorem 5.7.

Continuity result. If the measure graphs $\left(G_{n}, M_{n}\right)$ with uniform vertex degree bound converge weakly to the measure graph $(G, M)$, then the $Z_{\left(G_{n}, M_{n}\right)}$ converge to $Z_{(G, M)}$ compactly around zero.

This result covers all the earlier results on convergence of graph Zeta functions given in 6. 16, 19, 20]. It even strengthens them by providing an interpretation of the limit as the Zeta function of a graph. Even more, we derive the corresponding approximation for connected, finite graphs converging weakly in the sense of Benjamini-Schramm, cf. Theorem [5.9, Another new application is the convergence of the Ihara Zeta functions associated to sequences of percolated Cayley graphs with their probability laws $\mathbb{P}_{n}$ being weakly convergent, cf. Theorem 5.11.

Furthermore, the result can be used to provide a (rather large) class of new examples on which the Zeta function can be obtained via approximation. This is discussed in Section 6, where we study graphs allowing for a proper action of a sofic group with finite covolume. We explicitly construct a sequence of finite graphs converging towards the original graph as measure graphs. This is the content of Theorem 6.3. The convergence on the level of Zeta functions is immediate from the previous section.

The considerations of the present work are phrased within the measurable category. However, in prominent classes of examples we often have some additional topological information at hand. This and more will be addressed in a companion paper [22].

Acknowledgements. The authors gratefully acknowledge most inspiring discussions on Zeta functions with Anders Karlsson and Michel Lapidus and on non-commutative integration theory with Norbert Peyerimhoff, Peter Stollmann and Ivan Veselić. The authors thank Anton Deitmar for valuable remarks on groupoids and on the notation in the present manuscript. F.P. thanks Damien Gaboriau for a very enlightening discussion at the IAS at Hebrew University and for addressing the question of percolation measured graphs. Moreover, the authors would like to thank the referees for their careful reading of the manuscript and the corresponding suggestions. F.P. expresses his thanks for support through the German National Academic Foundation (Studienstiftung des deutschen Volkes). M.S. has been financially supported by the Graduiertenkolleg 1523/2 : Quantum and gravitational fields.

\section{LIST OF ESSENTIAL PIECES OF NOTATION}

Here we present a list of the main pieces of notation together with a short explanation and the number of the pages on which they are introduced.

- $G=(V, E)$ : graph with vertex set $V$ and edge set $E$ (Page 5 ).

- $V^{(2)}$ : set of pairs of vertices in the same connected component (Page 6).

- $a_{G}$ : the adjacency matrix of $G$ (Page 6)

- $B_{r}^{G}(x)$ rooted graph with root $x$ induced from the $r$-ball around $x$ (Page 6).

- $\mathcal{G}$ : groupoid (Page 9). 
- $\nu$ : transversal function on $\mathcal{G}$ (Page 9).

- $\eta$ : the canonical random variable assigning each vertex the mass one (Page 9).

- $\Omega$ units of the groupoid (Page 91).

- $\mathfrak{u}$ averaging function i.e. function satisfying $\nu * \mathfrak{u}=1$ (Page 12).

\section{THE FRAMEWORK OF MEASURE GRAPHS}

In this section we present the notation and concepts used throughout the paper. In particular, we introduce our concept of measure graphs over groupoids. These consists of a (not necessarily countable) measurable graph together with a measure satisfying some invariance property. The invariance property is phrased via a groupoid. More specifically, the basic pieces of data used in our setting are the following:

- A measurable graph $G=(V, E)$.

- A measurable groupoid $\mathcal{G}$ such that the graph is a space over the groupoid in the sense of Connes.

- A measure $M$ which is invariant with respect to the groupoid.

- an averaging function $\mathfrak{u}$ providing a connection between the groupoid and the graph.

These four pieces of data are discussed in the subsequent subsections.

1.1. Graphs. Here we introduce the concept of graphs used in the sequel. These will be undirected graphs with uniform bounded vertex degree and without loops.

By a graph we mean a tuple $G=(V, E)$ consisting of a set of vertices $V \neq \emptyset$ and a set of edges $E \subseteq V \times V$ such that the following holds:

- Whenever $(x, y)$ belongs to $E$ then so does $(y, x)$.

- There is no $x \in V$ such that $(x, x)$ belongs to $E$.

- There is a $D>0$ such that the cardinality of $\{y \in V:(x, y) \in E\}$ is bounded by $D$ for any $x \in V$.

Remark 1.1. We emphasize that we do not put any restrictions on the cardinality of $V$ nor $E$.

Let $G=(V, E)$ be a graph. For given $x \in V$ we call the pair $(G, x)$ a rooted graph with root $x$. If $(x, y)=e \in E$ we write $x \sim y$ and call $x=o(e)$ the origin and $y=t(e)$ the terminal vertex of $e$. For an edge $e=(o(e), t(e))$ we define the reversed edge via $\bar{e}=(t(e), o(e))$. Two edges $e, f$ are called incident if $\{t(e), o(e)\} \cap\{t(f), o(f)\}$ consists of exactly one element.

The vertex degree at $x$ is the number of edges with origin $x$. It will be denoted by $\operatorname{deg}(x)$. In this way, deg becomes a function from $V$ to the non-negative integers.

A path is a finite sequence of edges $\left(e_{1}, \ldots, e_{n}\right)$, such that $o\left(e_{i+1}\right)=t\left(e_{i}\right)$ for each $i=$ $1, \ldots, n-1$. The number of edges occurring in a path $P$ is called its length and is denoted by $\ell(P)$. Two vertices $x, y \in V$ are said to be connected, if there exists a path $\left(e_{1}, \ldots, e_{n}\right)$, such that $o\left(e_{1}\right)=x$ and $t\left(e_{n}\right)=y$. If $x, y \in V$ are connected their combinatorial distance, $d(x, y)$, is the length of the shortest path connecting them. If $x$ and $y$ are not connected we set $d(x, y)=\infty$.

A connected component in a graph is a maximal set of vertices such that the combinatorial distance between any two elements of this set is finite. For an $x \in V$ the connected component containing $x$ is the set $V(x)$ of all vertices which are connected with $x$. We denote the induced 
subgraph by $G(x)=(V(x), E \cap[V(x) \times V(x)])$. For vertices $x, y \in V$, we will write $x \approx y$ if $x$ and $y$ belong to the same connected component.

Further, for $r \in \mathbb{N}$, we let $B_{r}^{G}(x)$ denote the graph with root $x$ which is induced by $G$ when restricting the vertex set to the combinatorial $r$-ball around $x$. Note that by assumption on the uniform boundedness of the degree, $B_{r}^{G}(x)$ is finite and the graph $G(x)$ is at most countable.

The radius, $\varrho(G, x)$ of a finite connected graph $G$ with root $x$ is the maximal distance of a vertex from the root, i.e.

$$
\varrho(G, x)=\max \{d(y, x): y \in V(G)\} .
$$

Any graph comes naturally with a certain product space and a canonical function on it. This is discussed next. Let $G$ be a graph. Then, we define

$$
V^{(2)}:=V_{G}^{(2)}:=\{(x, y) \in V \times V: G(x)=G(y)\} \subset V \times V .
$$

On $V^{(2)}$ there is the canonical function, called adjacency matrix of $G$, defined via

$$
a_{G}: V^{(2)} \rightarrow\{0,1\}, a_{G}(x, y)=1 \text { if } x \sim y \text { and } a_{G}(x, y)=0 \text { else. }
$$

So, in particular, we have that

$$
E=a_{G}^{-1}(1) \subset V^{(2)} .
$$

We denote the restriction of $d$ to $V^{(2)}$ by $d$ again.

Whenever $V$ carries a $\sigma$-algebra, then $V^{(2)}$ becomes a measurable space with the $\sigma$-algebra induced by the product $\sigma$-algebra on $V \times V$ and so does its subset $E$.

The real numbers and the complex numbers (and subsets thereof) will always be equipped with the Borel- $\sigma$-algebra generated by the open subsets. Moreover, we will need the extended positive half-axis $[0, \infty]=[0, \infty) \cup\{\infty\}$. It will be equipped with the $\sigma$-algebra generated by the Borel- $\sigma$-algebra on $[0, \infty)$.

Two graphs $G_{1}=\left(V_{1}, E_{1}\right)$ and $G_{2}=\left(V_{2}, E_{2}\right)$ are called isomorphic if there exists a bijective map $\varphi: V_{1} \rightarrow V_{2}$ with $x \sim y$ if and only if $\varphi(x) \sim \varphi(y)$. This map is then called a graph isomorphism. Two finite rooted graphs are called isomorphic if there exists an isomorphism between them which maps the root of one into the root of the other graph. Obviously, isomorphy is an equivalence relation on all finite rooted graphs. For $r \geq 0$, we denote by $\mathcal{A}_{r}^{D}$ the set of all equivalence classes of finite connected rooted graphs with vertex degree bounded by $D$ and radius equal to $r$. By $\mathcal{A}^{D}$ we denote the union over $r \geq 0$ of all $\mathcal{A}_{r}^{D}$. Due to the boundedness assumption on the degree, this is a countable set. We will equip it with the discrete topology and the induced $\sigma$-algebra (both of which agree with the power set). For any $r \geq 0$ we let $\pi_{r}$ be the map

$$
\pi_{r}: V \rightarrow \mathcal{A}^{D}, x \mapsto\left[B_{r}^{G}(x)\right],
$$

where [.] denotes the class of a rooted graph modulo isomorphy. For a given $\alpha \in \mathcal{A}^{D}$ and a set of vertices $\widetilde{V} \subseteq V$ we let

$$
\widetilde{V}_{\alpha}:=\widetilde{V} \cap \pi_{\rho(\alpha)}^{-1}(\alpha)=\left\{x \in \widetilde{V}: \pi_{\rho(\alpha)}(x)=\alpha\right\}
$$

be the set of all vertices in $\widetilde{V}$ whose $\rho(\alpha)$-ball is isomorphic to $\alpha$. Here, of course, the choice $V=\widetilde{V}$ is possible resulting in the set $V_{\alpha}$.

If for a graph $G$ and $r \geq 0$, the subgraph of $G$ induced by the ball $B_{r}^{G}(x)$ around a vertex $x$ is a representative of the class $\alpha \in \mathcal{A}^{D}$, we will also write $B_{r}^{G}(x) \in \alpha$. 
We will be interested in graphs carrying a $\sigma$-algebra so that the graph is locally constant in a certain sense.

Definition 1.2 (Measurable graph). A pair $(G, \mathcal{B})$ consisting of a graph $G=(V, E)$ and a $\sigma$-algebra $\mathcal{B}$ on $V$ is called a measurable graph if the following conditions are satisfied:

- For any $r \geq 0$ the map $\pi_{r}: V \rightarrow \mathcal{A}^{D}, x \mapsto\left[B_{r}^{G}(x)\right]$, is measurable.

- The adjacency matrix $a_{G}: V^{(2)} \rightarrow\{0,1\}$ is measurable.

- For any two measurable $a, b: V^{(2)} \rightarrow[0, \infty]$, the matrix product

$$
a * b: V^{(2)} \rightarrow[0, \infty],(a * b)(x, y):=\sum_{z \approx x} a(x, z) b(z, y)
$$

is measurable.

Remark 1.3. We do not require the measurability of $V^{(2)}$ as a subset of $V \times V$ (equipped with the product $\sigma$-algebra). We rather work directly with the $\sigma$-algebra induced on $V^{(2)}$ by the product $\sigma$-algebra. While this does not play a role in the examples discussed below it may well be an advantage in further studies. In particular, it will be relevant in the already mentioned companion paper [22].

In a measurable graph certain basic quantities are automatically measurable. This is collected in the next proposition. It will be used tacitly in the sequel.

Proposition 1.4 (Measurability of basic quantities). Let $(G, \mathcal{B})$ be a measurable graph. Then, the following assertions hold:

(a) The combinatorial distance $d: V^{(2)} \rightarrow[0, \infty)$ is measurable.

(b) The diagonal $\{(x, x): x \in V\} \subset V^{(2)}$ is measurable.

(c) For any $r, s, t \geq 0$ and $\alpha, \beta \in \mathcal{A}^{D}$ the set

$$
\left\{(x, y) \in V^{(2)}: \pi_{r}(x)=\alpha, \pi_{s}(y)=\beta, d(x, y) \leq t\right\}
$$

is measurable.

Proof. Clearly, (b) is a direct consequence of (a). Similarly, (c) is a direct consequence of (a) and the measurability of the $\pi_{u}, u \geq 0$. Thus, it suffices to show (a): By measurability of the adjacency matrix $a_{G}$ and the matrix product all powers $a_{G}^{n}, n \in \mathbb{N}$, (defined inductively via $a_{G}^{1}:=a_{G}$ and $\left.a_{G}^{n+1}:=a_{G} * a_{G}^{n}\right)$ are measurable. Now, clearly, $d(x, y)=1$ holds if and only if $a_{G}(x, y)=1$ holds and, for $n \geq 1, d(x, y)=n$ holds if and only if both $a_{G}^{n}(x, y) \neq 0$ and $a_{G}^{k}(x, y)=0, k=1, \ldots, n-1$ hold. This shows measurability of the sets $\left\{(x, y) \in V^{(2)}\right.$ : $d(x, y)=n\}$ for $n \in \mathbb{N}$. This, then implies measurability of

$$
\left\{(x, y) \in V^{(2)}: d(x, y)=0\right\}=V^{(2)} \backslash \bigcup_{n \in \mathbb{N}}\left\{(x, y) \in V^{(2)}: d(x, y)=n\right\} .
$$

This finishes the proof.

Proposition 1.5 (Measurability of product of matrix with a function). Let $(G, \mathcal{B})$ be a measurable graph. Then, for any measurable $a: V^{(2)} \rightarrow[0, \infty]$ and any measurable $F: V \rightarrow$ $[0, \infty]$ the map

$$
a \widetilde{*} F: V \rightarrow[0, \infty], x \mapsto \sum_{y \approx x} a(x, y) F(y)
$$

is measurable. In particular, the vertex degree deg: $V \rightarrow[0, \infty)$ is measurable. 
Proof. As the $\sigma$-algebra on $V^{(2)}$ is the restriction of the product $\sigma$-algebra the maps

$$
p_{1}: V^{(2)} \rightarrow V,(x, y) \mapsto x, \text { and } j: V \rightarrow V^{(2)}, x \mapsto(x, x)
$$

are measurable. Hence,

$$
a \widetilde{*}=\left(a *\left(F \circ p_{1}\right)\right) \circ j
$$

is measurable as a composition of measurable functions. Now, the last statement follows from $\operatorname{deg}=a_{G} \widetilde{*} 1$.

The vertex set of a measurable graph can be disjointly decomposed into measurable sets of vertices whose neighborhood looks like a given isomorphism class $\alpha \in \mathcal{A}^{D}$. However, some care has to be taken when the connected component of a vertex is finite. To this end, for $r \geq 0$ we define

$$
V^{\mathrm{fin}, r}:=\{x \in V: \rho((G(x), x))=r\} .
$$

Proposition 1.6. Let $(G, \mathcal{B})$ be a measurable graph. For each $n \geq 0$ the vertex set $V$ can be written as

$$
V=\bigcup_{\alpha \in \mathcal{A}_{n}^{D}} V_{\alpha} \cup \bigcup_{r=0}^{n-1} V^{\mathrm{fin}, r}
$$

where the occurring sets are measurable and pairwise disjoint. Furthermore, for each $s \leq r$ the set $V^{\text {fin,r }}$ can be disjointly decomposed into

$$
V^{\mathrm{fin}, r}=\bigcup_{\alpha \in \mathcal{A}_{s}^{D}} V_{\alpha}^{\mathrm{fin}, r}
$$

where, of course, $V_{\alpha}^{\mathrm{fin}, r}=V^{\mathrm{fin}, r} \cap V_{\alpha}$.

Proof. All of the claimed properties follow easily from the measurability of the mappings $\pi_{r}$, $r \geq 0$, and the identity

$$
V^{\mathrm{fin}, r}=\bigcup_{\alpha \in \mathcal{A}_{r}^{D}} V_{\alpha} \backslash\left(\bigcup_{\beta \in \mathcal{A}_{r+1}^{D}} V_{\beta}\right)=\bigcup_{\alpha \in \mathcal{A}_{r}^{D}} \pi_{r}^{-1}(\alpha) \backslash\left(\bigcup_{\beta \in \mathcal{A}_{r+1}^{D}} \pi_{r+1}^{-1}(\beta)\right) .
$$

1.2. Graphs over groupoids and invariant measures. Here we discuss groupoids, invariant measures on groupoids and spaces over groupoids. In the measurable setting this can be found e.g. in Connes' lecture notes [8. Based on these lecture notes a discussion of random Schroedinger operators in this context was then given in 23]. There, a specific situation is singled out and studied in some detail. This situation is called 'admissible setting' there. Here, we basically present a graph version of the corresponding considerations centered around the admissible setting in 23 .

Notation. As usual we will denote the set of all measurable functions on a measurable space by $\mathcal{F}(X)$. The set of non-negative measurable functions is then denoted by $\mathcal{F}^{+}(X)$. The set of all measures on $X$ is denoted by $\mathcal{M}(X)$.

A concise definition of a groupoid is that it is a small category in which every morphism is an isomorphism. A more detailed definition can then be given as follows, see e.g. [31. 
Definition 1.7. A triple $\left(\mathcal{G}, \cdot,^{-1}\right)$ consisting of a set $\mathcal{G}$, a partially defined multiplication ·, and an inverse operation ${ }^{-1}: \mathcal{G} \rightarrow \mathcal{G}$ is called a groupoid if the following conditions are satisfied:

- $\left(g^{-1}\right)^{-1}=g$ for all $g \in \mathcal{G}$,

- If $g_{1} \cdot g_{2}$ and $g_{2} \cdot g_{3}$ exist, then $\left(g_{1} \cdot g_{2}\right) \cdot g_{3}$ and $g_{1} \cdot\left(g_{2} \cdot g_{3}\right)$ exist as well and they are equal,

- $g^{-1} \cdot g$ exists always and $g^{-1} \cdot g \cdot h=h$, whenever $g \cdot h$ exists,

- $h \cdot h^{-1}$ exists always and $g \cdot h \cdot h^{-1}=g$, whenever $g \cdot h$ exists.

A given groupoid $\mathcal{G}$ comes along with the following standard objects. The subset

$$
\Omega:=\mathcal{G}^{(0)}:=\left\{g \cdot g^{-1} \mid g \in \mathcal{G}\right\}
$$

is called the set of units.

For $g \in \mathcal{G}$ we define its range $r(g)$ by $r(g)=g \cdot g^{-1}$ and its source by $s(g)=g^{-1} \cdot g$. Moreover, we set $\mathcal{G}^{\omega}=r^{-1}(\{\omega\})$ for any unit $\omega \in \mathcal{G}^{(0)}$. One easily checks that $g \cdot h$ exists if and only if $r(h)=s(g)$.

The groupoids under consideration will always be measurable, i.e., they possess $\sigma$-algebras such that all relevant maps are measurable. More precisely, we require that $\cdot: \mathcal{G}_{G}^{(2)} \rightarrow \mathcal{G}$, ${ }^{-1}: \mathcal{G} \rightarrow \mathcal{G}, s, r: \mathcal{G} \rightarrow \mathcal{G}^{(0)}$ are measurable, where

$$
\mathcal{G}_{G}^{(2)}:=\left\{\left(g_{1}, g_{2}\right) \mid s\left(g_{1}\right)=r\left(g_{2}\right)\right\} \subset \mathcal{G}^{2}
$$

and $\mathcal{G}^{(0)} \subset \mathcal{G}$ are equipped with the induced $\sigma$-algebras. Furthermore, we assume that singletons $\{\omega\}$ with $\omega \in \mathcal{G}^{(0)}$ are measurable as subsets of $\mathcal{G}^{(0)}$. In this way, $\mathcal{G}^{\omega} \subset \mathcal{G}$ become measurable sets (and thus measurable spaces).

Definition 1.8 (Graph over $\mathcal{G}$ ). Let $\mathcal{G}$ be a measurable groupoid with the previously introduced notations. A triple $(G, \pi, J)$ consisting of a measurable graph $G$ with vertex set $V$ and maps $\pi$ and $J$ is called a graph over $\mathcal{G}$ if the following properties are satisfied.

- The map $\pi: V \rightarrow \Omega$ is measurable.

- For any $\omega \in \Omega$ the induced graph $G^{\omega}$ on the vertex set

$$
V^{\omega}:=\pi^{-1}(\{\omega\})
$$

is countable.

- The map $\eta: \Omega \rightarrow \mathcal{M}(V), \eta^{\omega}:=\sum_{y \in \pi^{-1}(\omega)} \delta_{y}$, is measurable in the sense that for any measurable $F: V \rightarrow[0, \infty]$, the map $\Omega \rightarrow[0, \infty], \omega \mapsto \eta^{\omega}(F)$, is measurable.

- The map $J$ assigns, to every $g \in \mathcal{G}$, a graph isomorphism $J(g): G^{s(g)} \rightarrow G^{r(g)}$ with the properties $J\left(g^{-1}\right)=J(g)^{-1}$ and $J\left(g_{1} \cdot g_{2}\right)=J\left(g_{1}\right) \circ J\left(g_{2}\right)$ if $s\left(g_{1}\right)=r\left(g_{2}\right)$.

The map $\eta$ is called the canonical random variable.

Notation. To simplify notation, we will often write $g h$ respectively $g x$ for $g \cdot h$ respectively $J(g) x$.

Our next aim is to exhibit natural measures on these objects. The first step in this direction is the definition of a transverse function.

Definition 1.9 (Transversal function). Let $\mathcal{G}$ be a measurable groupoid and with the notation given above. A transversal function $\nu$ of $\mathcal{G}$ is a map $\nu: \Omega \rightarrow \mathcal{M}(\mathcal{G})$ with the following properties: 
- The map $\omega \mapsto \nu^{\omega}(f)$ is measurable for every $f \in \mathcal{F}^{+}(\mathcal{G})$.

- $\nu^{\omega}$ is supported on $\mathcal{G}^{\omega}$, i.e., $\nu^{\omega}\left(\mathcal{G}-\mathcal{G}^{\omega}\right)=0$.

- $\nu$ satisfies the following invariance condition

$$
\int_{\mathcal{G}^{s(g)}} f(g \cdot h) d \nu^{s(g)}(h)=\int_{\mathcal{G}^{r(g)}} f(k) d \nu^{r(g)}(k)
$$

for all $g \in \mathcal{G}$ and $f \in \mathcal{F}^{+}\left(\mathcal{G}^{r(g)}\right)$.

In the examples which will be considered later in the present paper, the measures $\nu^{\omega}$ will be simple counting measures defined on countable fibers $r^{-1}(\omega)$.

In the next definition we introduce appropriate measures on the base space $\Omega$ of an abstract groupoid $\mathcal{G}$.

Definition 1.10 (Invariant measure). Let $\mathcal{G}$ be a measurable groupoid with a transversal function $\nu$. A measure $m$ on the base space $\left(\Omega, \mathcal{B}_{\Omega}\right)$ of units is called $\nu$-invariant (or simply invariant, if there is no ambiguity in the choice of $\nu$ ) if

$$
m \circ \nu=(m \circ \nu)^{\sim},
$$

where $m \circ \nu$ is the measure on $\mathcal{G}$ defined by $(m \circ \nu)(f)=\int_{\Omega} \nu^{\omega}(f) d m(\omega)$ for measurable $f: \mathcal{G} \longrightarrow[0, \infty]$ and $(m \circ \nu)^{\sim}(f)=(m \circ \nu)(\tilde{f})$ with $\tilde{f}(g)=f\left(g^{-1}\right)$.

Analogously to transversal functions on the groupoid, we introduce a corresponding fiberwise consistent family $\alpha$ of measures on a graph over a groupoid.

Definition 1.11 (Random variable in the sense of Connes). Let $\mathcal{G}$ be a measurable groupoid and $G$ a graph over $\mathcal{G}$ with vertex set $V$. A choice of measures $\xi: \Omega \rightarrow \mathcal{M}(V)$ is called a random variable with values in $G$ (in the sense of Connes) if it has the following properties:

- The map $\omega \mapsto \xi^{\omega}(f)$ is measurable for every $f \in \mathcal{F}^{+}(V)$,

- $\xi^{\omega}$ is supported on $G^{\omega}$, i.e., $\xi^{\omega}\left(V-V^{\omega}\right)=0$,

- $\xi$ satisfies the following invariance condition

$$
\int_{V^{s(g)}} f(J(g) x) d \xi^{s(g)}(x)=\int_{V^{r(g)}} f(y) d \xi^{r(g)}(y)
$$

for all $g \in \mathcal{G}$ and $f \in \mathcal{F}^{+}\left(V^{r(g)}\right)$.

Remark 1.12. Let $\xi$ be a random variable.

- By considering (positive) linear combinations of functions of the form

$$
F: V^{2} \rightarrow[0, \infty],(x, y) \mapsto f(x) g(y),
$$

with measurable $f, g: V \rightarrow[0, \infty]$ and using standard monotone class arguments, we can easily obtain measurability of

$$
V \times \Omega \rightarrow[0, \infty],(x, \omega) \rightarrow \xi^{\omega}(F(x, \cdot)),
$$

for any measurable $F: V \times V \rightarrow[0, \infty]$.

- If furthermore $V^{(2)}$ is a measurable subset of $V \times V$, then any measurable function $F$ on $V^{(2)} \rightarrow[0, \infty]$ can be extended (by zero) to a measurable function on $V \times V$. Thus, in this case we obtain measurability of $V \times \Omega \rightarrow[0, \infty],(x, \omega) \rightarrow \xi^{\omega}(F(x, \cdot))$ for any measurable $F: V^{(2)} \rightarrow[0, \infty]$.

We can actually provide a large supply of random variables. 
Proposition 1.13 (Generating random variables). Let $\mathcal{G}$ be a measurable groupoid and $G$ a graph over $\mathcal{G}$ with vertex set $V$. Then, the canonical random variable $\eta$ is a random variable. Moreover, for any measurable $H: V \rightarrow[0, \infty]$ with $H(g x)=H(x)$ for all $x \in V$ and $g \in \mathcal{G}$ with $\pi(x)=s(g)$ the map

$$
\xi_{H}: \Omega \rightarrow \mathcal{M}(V), \xi_{H}^{\omega}:=\sum_{y \in \pi^{-1}(\omega)} H(y) \delta_{y}
$$

is a random variable.

Proof. The canonical random variable $\eta$ is a random variable by the very definition of a graph over a groupoid. Now, consider a measurable $H: V \rightarrow[0, \infty]$. We have to show a measurability and an invariance property of $\xi_{H}$. Now, whenever $F: V \rightarrow[0, \infty]$ is measurable, then so is $F H: V \rightarrow[0, \infty]$. Hence,

$$
\omega \mapsto \xi_{H}^{\omega}=\eta^{\omega}(H F)
$$

is measurable by the assumption on $\eta$. The invariance of $\xi_{H}$ is clear from the invariance property of $H$.

Remark 1.14. If the diagonal $\{(x, x): x \in V\}$ is a measurable subset of $V \times V$, then any random variable arises in this way. Indeed, in the case the previous remark applied with $F$ the characteristic function of the diagonal, gives easily that for any random variable $\xi$ the function $H(x)=\xi^{\pi(x)}(F(x, \cdot))$ is measurable and invariant. By construction $H$ is the density of $\xi$ with respect to $\eta$.

Whenever $G$ is a graph over a groupoid $\mathcal{G}$ with transverse $\nu$, we use the following notation for the convolution of a $w \in \mathcal{F}^{+}(V)$ with respect to $\nu$

$$
\nu * w(x):=\int_{\mathcal{G}^{\pi(x)}} w\left(g^{-1} x\right) d \nu^{\pi(x)}(g) \quad \text { for } x \in V .
$$

A crucial fact about the integration of random variables is given in the following lemma, essentially contained in (the proof of) Lemma III.1 in [8], see Lemma 2.9 of [23] as well.

Lemma 1.15. Let $\mathcal{G}$ be a measurable groupoid with transversal function $\nu$ and $\nu$-invariant measure $m$. Let $G$ be a graph over $\mathcal{G}$.

(a) The integral $\int_{\Omega} \nu^{\omega}(f) d m(\omega)$ does not depend on $f \in \mathcal{F}^{+}(\mathcal{G})$, provided $f$ satisfies $\nu(\tilde{f}) \equiv 1$.

(b) For a given random variable $\xi$ with values in $G$ the integral $\int_{\Omega} \xi^{\omega}(\mathfrak{u}) d m(\omega)$ does not depend on $\mathfrak{u}$, provided $\mathfrak{u} \in \mathcal{F}^{+}(V)$ satisfies $\nu * \mathfrak{u} \equiv 1$.

The previous lemma gives the possibility to define an integral over a random variable.

Definition 1.16 (Integral of a random variable). Let $\mathcal{G}$ be a measurable groupoid with transversal function $\nu$ and $\nu$-invariant measure $m$. Let $G$ be a graph over $\mathcal{G}$ with vertex set $V$ such that there exists a $\mathfrak{u} \in \mathcal{F}^{+}(V)$ satisfying $\nu * \mathfrak{u} \equiv 1$. Then, the integral over the random variable $\xi$ is denoted as $\int \xi$ and defined via

$$
\int \xi=\int_{\Omega} \xi^{\omega}(\mathfrak{u}) d m(\omega)
$$

where $\mathfrak{u}$ is any (not necessarily strictly positive) element of $\mathcal{F}^{+}(V)$ satisfying $\nu * \mathfrak{u} \equiv 1$.

The functions $\mathfrak{u}$ appearing in the previous definition deserve a special name. 
Definition 1.17 (Averaging function). Let $\mathcal{G}$ be a measurable groupoid with transversal function $\nu$ and $\nu$-invariant measure $m$. Let $G$ be a graph over $\mathcal{G}$ with vertex set $V$. Then, any function $\mathfrak{u} \in \mathcal{F}^{+}(V)$ satisfying $\nu * \mathfrak{u} \equiv 1$ is called an averaging function.

We note that existence of an averaging function is quite essential to our approach. In fact, it is only via these averaging functions that the integration of random variables could be defined above. On the conceptual level the existence of an averaging function $\mathfrak{u}$ can be understood as giving a way to relate $\mathcal{G}$ and $G$ via a map from $\mathcal{F}(\mathcal{G})$ to $\mathcal{F}(V)$. Namely, every averaging function $\mathfrak{u}$ gives rise to the fiberwise defined map

$$
q=q_{\mathfrak{u}}: \mathcal{F}(\mathcal{G}) \rightarrow \mathcal{F}(V)
$$

with

$$
q(f)(x):=\int_{\mathcal{G}^{\pi(x)}} \mathfrak{u}\left(g^{-1} x\right) f(g) d \nu^{\pi(x)}(g)
$$

for all $x \in V$. Note the defining property of $\mathfrak{u}$ implies that the map $q$ satisfies $q\left(1_{\mathcal{G}}\right)=1_{V}$ (see Section 2 of 23 for further discussion).

1.3. Measure graphs. After all these preparations we can now introduce the main concept of our study. We will need one further assumption on countability of generators of the $\sigma$ algebras in question in order to apply the integration theory developed in [8].

Definition 1.18 (Measure graph over a groupoid). A pair $(G, M)$ consisting of a measurable graph $G$ and a measure $M$ on the vertices of $G$ is called a measure graph over the groupoid $\mathcal{G}$ or just measure graph for short if the following properties hold:

- $\mathcal{G}$ is a measurable groupoid and $G$ is a graph over $\mathcal{G}$.

- $\mathcal{G}$ admits a transversal function $\nu$ together with an invariant measure $m$ and $M=m \circ \eta$ (where $\eta$ is the canonical random variable).

- The $\sigma$-algebras of both $V$ and $\Omega$ possess a countable basis of sets, all of which have finite measure w.r.t. $M$ (respectively w.r.t. $m$ ).

- There exists a strictly positive averaging function $\mathfrak{u}$.

- The canonical random variable $\eta$ is integrable, i.e. $\int \eta=\int_{\Omega} \eta^{\omega}(\mathfrak{u}) d m(\omega)=$ $\int_{V} \mathfrak{u}(x) d M(x)<\infty$.

The measure $m$ will be called the invariant measure underlying the measure $M$ and $\nu$ will be called the underlying transversal on the groupoid.

Remark 1.19. The above assumptions ensure that our setup falls within the general framework of [8] (as specified in Definition 2.6 of [23]). This will enable us to use the associated von Neumann algebras and to use integration theory. The countability assumptions on the $\sigma$-algebras mean that the condition of being 'propre' in the sense of [8] is satisfied. Integrability of the canonical random variable will yield a $\sigma$-finite weight (even a trace) on the corresponding von Neumann algebra and strict positivity of the averaging function will entail faithfulness of this weight.

For our further considerations two features of measure graphs will be crucial (see below):

- Any measure graph comes with a natural Hilbert space $L^{2}(V, M)$. We will be concerned with operators on this Hilbert space.

- Any measure graph naturally allows for integration of random variable as it possesses by its very definition an averaging function. 
1.4. Some examples of measure graphs. In this section we discuss some examples of measure graphs over groupoids. In particular, we discuss the integration of their random variables.

1.4.1. Finite graphs. Let $G$ be a finite connected graph with vertex set $V$. Equip $V$ with the discrete $\sigma$-algebra, which is finite. Let $\mathcal{G}$ be the trivial groupoid (consisting only of one element $e$ ) acting on $G$ as the identity. Its set of units $\Omega=\{e\}$ consists of only one point and $\pi: V \rightarrow\{e\}$ is measurable. Let $\nu$ be the measure giving value 1 to the point $\{e\}$. Then, the measure $m$ giving value $1 / C$ to the point $\{e\}$ is invariant and the constant function $\mathfrak{u}=1$ satisfies $\nu * \mathfrak{u} \equiv 1$. The measure $M=m \circ \eta$ on $V$ is the counting measure on the vertex set normalized by $1 / C$. In this way, $(G, M)$ is a measure graph over $\mathcal{G}$. Furthermore, all random variables are given by functions on the vertex set and their integral equals the sum of their values normalized by $1 / C$.

1.4.2. Periodic graphs. We first recall the notion of a periodic graph.

Definition 1.20 (Periodic graph). A pair $(G, \Gamma)$ consisting of a countable infinite connected graph $G=(V, E)$ and a countable subgroup $\Gamma$ of the automorphism group of $G$ is called periodic graph, if

- $\Gamma$ acts on $V$ properly, i.e., for each $x \in V$ the stabilizer $\Gamma_{x}=\{\gamma \in \Gamma: \gamma x=x\}$ is finite.

- $\Gamma$ acts on $V$ with finite co-volume, i.e., there is a set $F \subset \Gamma$ containing exactly one representative of each of the classes of $V / \Gamma$ satisfying $\sum_{f \in F} \frac{1}{\left|\Gamma_{f}\right|}<\infty$.

The subset $F$ is called a fundamental domain.

Let $(G, \Gamma)$ be a periodic graph. We equip its vertex set $V$ with the discrete $\sigma$-algebra, which is countably generated. Let $\mathcal{G}$ be given by $\Gamma$ with the corresponding action on $G$. The space of units $\Omega$ consists exactly of the neutral element $e$ of $\Gamma$ and the map $\pi: V \rightarrow\{e\}$ is clearly measurable. A transversal function $\nu=\nu^{e}$ is given by the counting measure on $\Gamma$. The measure $m$ giving mass 1 to the set $\{e\}$ is invariant. Fix a fundamental domain $F$ of finite co-volume of the action of $\Gamma$ and define the function $\mathfrak{u}_{0}$ on $V$ by

$$
\mathfrak{u}_{0}(x):=\frac{1}{\left|\Gamma_{x}\right|} 1_{F}(x) .
$$

Here $\left|\Gamma_{x}\right|$ denotes the number of elements of the stabilizer of $x$ and $1_{F}$ is the characteristic function of $F$. A short calculation shows that $\nu * \mathfrak{u}_{0}(x)=1$ for all $x \in V$, i.e., $\mathfrak{u}_{0}$ is an averaging function. It is not strictly positive, though. However, for any $\gamma \in \Gamma$, the function $\mathfrak{u}_{\gamma}(\cdot):=\mathfrak{u}_{0}(\gamma \cdot)$ is an averaging function as well. So, if we chose a sequence $\left(c_{\gamma}\right)_{\gamma \in \Gamma}$ of positive numbers with $\sum c_{\gamma}=1$, then

$$
\mathfrak{u}:=\sum_{\gamma \in \Gamma} c_{\gamma} \mathfrak{u}_{\gamma}
$$

will be a strictly positive averaging function. The measure $M=m \circ \eta$ is just the counting measure on the vertex set. In this way, $(G, M)$ is a measure graph over $\mathcal{G}=\Gamma$.

Recall the definition of the random variable $\xi_{F}$ for a $\Gamma$-invariant function $F: V \rightarrow[0, \infty]$ (see Lemma 1.13). By Lemma 1.15its integral satisfies

$$
\int \xi_{F}=\int_{V} F \mathfrak{u} \mathrm{d} M=\int_{V} F \mathfrak{u}_{0} \mathrm{~d} M=\sum_{x \in F} \frac{F(x)}{\left|\Gamma_{x}\right|} .
$$


1.4.3. Percolation graphs. Let $\Gamma$ be a finitely generated group with a symmetric set of generators $I=\left\{g_{1}, \ldots, g_{l}, g_{1}^{-1}, \ldots, g_{l}^{-1}\right\}$ and let $e$ be its neutral element. By $G_{0}$ we denote the corresponding right Cayley graph, i.e., $\gamma, \gamma^{\prime} \in \Gamma$ are neighbors in $G_{0}$ if and only if there exists $g \in I$ such that $\gamma=\gamma^{\prime} g$. The left-action of $\Gamma$ on itself induces graph isomorphisms of $G_{0}$. We perform vertex percolation on $G_{0}$ and construct a measure graph from its realizations. We equip the space

$$
\Theta:=\{0,1\}^{\Gamma}
$$

with the product $\sigma$-algebra (which is countably generated) and define the shift-action of $\Gamma$ by

$$
\Gamma \times \Theta \rightarrow \Theta,(\gamma, \theta) \mapsto \gamma \theta:=\theta\left(\gamma^{-1} \cdot\right) .
$$

Each element $\theta \in \Theta$ is identified with the graph $G_{\theta}=\left(\Gamma, E_{\theta}\right)$ whose edge set is

$$
E_{\theta}=\left\{\left(\gamma, \gamma^{\prime}\right) \in \Gamma \times \Gamma: \gamma \sim \gamma \text { in } G_{0} \text { and } \theta(\gamma)=\theta\left(\gamma^{\prime}\right)=1\right\} .
$$

In other words, $G_{\theta}$ is obtained from $G_{0}$ by deleting all edges that are adjacent to a vertex $\gamma \in \Gamma$ which satisfies $\theta(\gamma)=0$.

The vertex set of the percolation measure graph is defined by $V:=\Gamma \times \Theta$ and equipped with the product $\sigma$-algebra (here $\Gamma$ carries the discrete $\sigma$-algebra). We say that $(\gamma, \theta)$ and $\left(\gamma^{\prime}, \theta^{\prime}\right)$ are adjacent if and only if $\theta=\theta^{\prime}$ and $\gamma \sim \gamma^{\prime}$ in $G_{\theta}$. The so obtained graph is denoted by $G_{\Gamma, \text { perc }}$.

Proposition 1.21. $G_{\Gamma, \text { perc }}$ is a measurable graph.

Proof. We first show that $\pi_{r}: V \rightarrow \mathcal{A}^{D}$ is measurable. For $\alpha \in \mathcal{A}^{D}$ we obtain

$$
\begin{aligned}
\pi_{r}^{-1}(\alpha) & =\left\{(\gamma, \theta) \in \Gamma \times \Theta: B_{r}^{G_{\Gamma, \text { perc }}}((\gamma, \theta)) \in \alpha\right\} \\
& =\left\{(\gamma, \theta) \in \Gamma \times \Theta: B_{r}^{G_{\theta}}(\gamma) \in \alpha\right\} \\
& =\left\{(\gamma, \theta) \in \Gamma \times \Theta: B_{r}^{G_{\gamma}-1 \theta}(e) \in \alpha\right\} \\
& =\bigcup_{\gamma \in \Gamma}\left[\{\gamma\} \times \gamma\left\{\theta \in \Theta: B_{r}^{G_{\theta}}(e) \in \alpha\right\}\right] .
\end{aligned}
$$

The set $\left\{\theta \in \Theta: B_{r}^{G_{\theta}}(e) \in \alpha\right\}$ is a finite union of cylinder sets in $\Theta$. In particular, it is measurable. Since the $\Gamma$-action on $\Theta$ is also measurable we obtain the measurability of $\pi_{r}^{-1}(\alpha)$.

The measurability of the adjacency matrix on $V^{(2)}$ is a consequence of

$$
\begin{aligned}
a_{G_{\Gamma, \text { perc }}}^{-1}(\{1\}) & =\left\{\left((\gamma, \theta),\left(\gamma^{\prime}, \theta^{\prime}\right)\right) \in V^{(2)}: \theta=\theta^{\prime} \text { and } \gamma \sim \gamma^{\prime} \text { in } G_{\theta}\right\} \\
& =V^{(2)} \cap\left\{\left((\gamma, \theta),\left(\gamma^{\prime}, \theta^{\prime}\right)\right) \in V \times V \text { : there ex. } g \in I \text { s.t. } \gamma^{\prime}=\gamma g\right\} \\
& =V^{(2)} \cap \bigcup_{(\gamma, g) \in \Gamma \times I}[(\{\gamma\} \times \Theta) \times(\{\gamma g\} \times \Theta)] .
\end{aligned}
$$

Here we have used the following two facts.

- $\left((\gamma, \theta),\left(\gamma^{\prime}, \theta^{\prime}\right)\right) \in V^{(2)}$ implies $\theta=\theta^{\prime}$,

- if $\gamma$ and $\gamma^{\prime}$ belong to the same connected component in $G_{\theta}$ and satisfy $\gamma^{\prime}=\gamma g$ for some $g \in I$ then $\gamma \sim \gamma^{\prime}$ in $G_{\theta}$. 
It remains to show the measurability of the matrix products on $V^{(2)}$. To this end, let measurable $a, b: V^{(2)} \rightarrow[0, \infty]$ be given. For fixed $\gamma \in \Gamma$ we define

$$
a_{\gamma}: V^{(2)} \rightarrow[0, \infty],\left(\left(\gamma^{\prime}, \theta\right),\left(\gamma^{\prime \prime}, \theta\right)\right) \mapsto \begin{cases}a\left(\left(\left(\gamma^{\prime}, \theta\right),(\gamma, \theta)\right)\right) & \text { if }\left(\gamma^{\prime}, \theta\right) \approx(\gamma, \theta) \\ 0 & \text { else }\end{cases}
$$

and

$$
b^{\gamma}: V^{(2)} \rightarrow[0, \infty],\left(\left(\gamma^{\prime}, \theta\right),\left(\gamma^{\prime \prime}, \theta\right)\right) \mapsto \begin{cases}b\left(\left((\gamma, \theta),\left(\gamma^{\prime \prime}, \theta\right)\right)\right) & \text { if }\left(\gamma^{\prime \prime}, \theta\right) \approx(\gamma, \theta) \\ 0 & \text { else }\end{cases}
$$

These functions satisfy

$$
a * b=\sum_{\gamma \in \Gamma} a_{\gamma} b^{\gamma}
$$

Therefore, it suffices to show the measurability of $a_{\gamma}$ and $b^{\gamma}$. Let $I=(C, \infty]$ with $C \in[0, \infty]$. We obtain

$$
\begin{aligned}
a_{\gamma}^{-1}(I) & =a^{-1}(I) \cap\left\{\left(\left(\gamma^{\prime}, \theta\right),(\gamma, \theta)\right):\left(\gamma^{\prime}, \theta\right) \in \Gamma \times \Theta \text { and }\left(\gamma^{\prime}, \theta\right) \approx(\gamma, \theta)\right\} \\
& =a^{-1}(I) \cap \bigcup_{\gamma^{\prime} \in \Gamma}\left[\left(\left\{\gamma^{\prime}\right\} \times \Theta\right) \times(\{\gamma\} \times \Theta)\right] .
\end{aligned}
$$

For the last identity we used $a^{-1}(I) \subseteq V^{(2)}$ and

$$
\begin{aligned}
& \left\{\left(\left(\gamma^{\prime}, \theta\right),(\gamma, \theta)\right):\left(\gamma^{\prime}, \theta\right) \in \Gamma \times \Theta \text { and }\left(\gamma^{\prime}, \theta\right) \approx(\gamma, \theta)\right\} \\
& =V^{(2)} \cap \bigcup_{\gamma^{\prime} \in \Gamma}\left[\left(\left\{\gamma^{\prime}\right\} \times \Theta\right) \times(\{\gamma\} \times \Theta)\right] .
\end{aligned}
$$

This shows the measurability of $a_{\gamma}$ and a similar reasoning yields the measurability of $b^{\gamma}$.

The groupoid which acts upon $G_{\Gamma \text {, perc }}$ is the semidirect product $\Gamma \ltimes \Theta$ with respect to the shift-action of $\Gamma$. More precisely, we let $\mathcal{G}=\Gamma \times \Theta$ and define a partially defined multiplication on $\mathcal{G}$ by

$$
(\gamma, \theta) \cdot\left(\gamma^{\prime}, \theta^{\prime}\right)=\left(\gamma \gamma^{\prime}, \theta\right) \text { iff } \theta=\gamma \theta^{\prime}
$$

and an inverse operation by

$$
(\gamma, \theta)^{-1}=\left(\gamma^{-1}, \gamma^{-1} \theta\right)
$$

The set of units $\Omega=\mathcal{G}^{(0)}=\{(e, \theta): \theta \in \Theta\}$ is tacitly identified with $\Theta$ in the obvious manner.

We now give $G_{\Gamma \text {,perc }}$ the structure of a graph over $\mathcal{G}$. The mapping $\pi: V \rightarrow \Theta,(\gamma, \theta) \mapsto \theta$ is clearly measurable and the canonical random variable $\eta$ is measurable as well. As a set, $\mathcal{G}$ coincides with the vertex set $V$ of the graph $G_{\Gamma \text {,perc }}$. Hence, the groupoid acts naturally upon $V$ by left multiplication. The induced graph on the fiber $\pi^{-1}(\{\theta\})$ is isomorphic to $G_{\theta}$. Since left-multiplication with $\gamma$ induces a graph isomorphism $G_{\gamma^{-1} \theta} \rightarrow G_{\theta}$ the groupoid acts by graph isomorphisms between the fibers of $\pi$. Therefore, $G_{\Gamma, \text { perc }}$ is a graph over $\mathcal{G}$.

A transversal function of $\mathcal{G}$ is

$$
\nu: \Theta \rightarrow \mathcal{M}(\mathcal{G}), \nu^{\theta}:=\sum_{\gamma \in \Gamma} \delta_{(\gamma, \theta)} .
$$

It can easily be checked that any probability measure $\mathbb{P}$ on $\Theta$ which is invariant with respect to the $\Gamma$-shift is invariant with respect to $\nu$, see [8, Cor. II.7] as well. We fix such a measure and let $M_{\mathbb{P}}=\mathbb{P} \circ \eta$. For any $\gamma \in \Gamma$ the function $\mathfrak{u}_{\gamma}:=1_{\{\gamma\} \times \Theta}$ is an averaging function. 
Taking suitable linear combinations yields a strictly positive averaging function $\mathfrak{u}$. In this way, $\left(G_{\Gamma, \text { perc }}, M_{\mathbb{P}}\right)$ is a measure graph over $\mathcal{G}$.

Recall the definition of the random variable $\xi_{F}$ for a $\mathcal{G}$-invariant function $F: V \rightarrow[0, \infty]$ (see Lemma 1.13). By Lemma 1.15 its integral satisfies

$$
\int \xi_{F}=\int_{V} F \mathfrak{u} \mathrm{d} M_{\mathbb{P}}=\int_{V} F \mathfrak{u}_{e} \mathrm{~d} M_{\mathbb{P}}=\int_{\Theta} F((e, \theta)) \mathrm{d} \mathbb{P} .
$$

1.4.4. Graphings. Graphings have attracted quite some attention. In particular, weakly convergent graph sequences can be shown to have graphings as limits (see e.g. [12]). As discussed next, they also fall within our framework.

Recall that a graphing, $\mathcal{X}=\left(X, \mu, \iota_{1}, \ldots, \iota_{N}\right)$, consists of a compact metric space $X$ equipped with the Borel- $\sigma$-algebra together with continuous involutions $\iota_{1}, \ldots, \iota_{N}: X \rightarrow X$ and a Borel probability measure $\mu$ on $X$ which is invariant under the $\iota_{j}, j=1, \ldots, N$.

To a given graphing $\mathcal{X}$ we associate a measure graph. Let $\mathcal{J}:=\left\langle\iota_{1}, \ldots, \iota_{n}\right\rangle$ be the group generated by the involutions. By $\mathcal{R}(\mathcal{X})$ we denote the induced equivalence relation, that is,

$$
\mathcal{R}(\mathcal{X})=\{(x, y) \in X \times X: \text { there ex. } \iota \in \mathcal{J} \text { s.t. } x=\iota y\} .
$$

It is equipped with the induced $\sigma$-algebra (it is countably generated since the $\sigma$-algebra of $X$ is countably generated). The vertex set of the graph associated with $\mathcal{X}$ is $V_{\mathcal{X}}:=\mathcal{R}(\mathcal{X})$. We say that two vertices $(x, y),(w, z) \in V_{\mathcal{X}}$ are connected if and only if $w=x$ and $y=\iota_{j} z$ with $\iota_{j} z \neq z$ for some $1 \leq j \leq n$. The so constructed graph is denoted by $G_{\mathcal{X}}$.

Proposition 1.22. $G_{\mathcal{X}}$ is a measurable graph.

Proof. The proof of the measurability conditions can be shown in a similar way as for the percolation graphs considered above.

The equivalence relation $\mathcal{R}(\mathcal{X})$ is a groupoid with partially defined multiplication

$$
(x, y) \cdot(w, z)=(x, z) \text { iff } w=y
$$

and inverse operation

$$
(x, y)^{-1}=(y, x) .
$$

As measurable space the set of units $\Omega=\mathcal{R}(\mathcal{X})^{(0)}=\{(x, x): x \in X\}$ is equivalent to the Borel space $X$. In the following we tacitly identify both.

We now give $G_{\mathcal{X}}$ the structure of a graph over $\mathcal{R}(\mathcal{X})$. The map $\pi: V_{\mathcal{X}} \rightarrow X,(x, y) \mapsto x$ is clearly measurable. Moreover, the following holds.

Proposition 1.23. The canonical random variable $\eta$ is measurable.

Proof. Let $\mathcal{J}$ be the group generated by the $\iota_{j}, j=1, \ldots, n$. Note that by continuity of the action for arbitrary $\gamma, \varrho \in \mathcal{J}$ the set $\{x \in X: \gamma(x) \neq \varrho(x)\}$ is open and its complement is closed. So, both sets are measurable. Let now $\gamma_{1}, \gamma_{2}, \ldots$, be an enumeration of $\mathcal{J}$. By induction we can then construct for any natural number $n$ a decomposition of $X$ into disjoint measurable sets $X_{n, 1}, \ldots, X_{n, k_{n}}$, such that for arbitrary fixed $\gamma, \varrho \in\left\{\gamma_{1}, \ldots, \gamma_{n}\right\}$ and $j \in$ $\left\{1, \ldots, k_{n}\right\}$ we either have $\gamma x=\varrho x$ for all $x \in X_{n, j}$ or $\gamma x \neq \varrho x$ for all $x \in X_{n, j}$. Let then for any natural number $n$ and any $j \in\left\{1, \ldots, k_{n}\right\}$ be $\mathcal{J}_{n, j}$ a maximal subset of $\left\{\gamma_{1}, \ldots, \gamma_{n}\right\}$ with 
$\gamma(x) \neq \varrho(x)$ for all $x \in X_{n, j}$ and $\gamma, \varrho \in \mathcal{J}_{n, j}$ with $\gamma \neq \varrho$. Denote the characteristic function of $X_{n, j}$ by $1_{X_{n, j}}$. Then, for any measurable nonnegative $F$ the function

$$
X \longrightarrow[0, \infty), x \mapsto \eta_{n}^{x}(F):=\sum_{j=1}^{k_{n}} 1_{X_{n, j}}(x)\left(\sum_{\gamma \in \mathcal{J}_{n, j}} F(x, \gamma x)\right),
$$

is measurable (as $F$ is measurable and the $X_{n, j}$ are measurable). Moreover, by maximality of the $\mathcal{J}_{n, j}$ we have $\eta_{n}(F) \rightarrow \eta(F)$ pointwise. This is the desired measurability of $\eta$.

As a set, $\mathcal{R}(\mathcal{X})$ coincides with the vertex set $V_{\mathcal{X}}$. Hence, the groupoid acts naturally upon $V_{\mathcal{X}}$ by left multiplication. The fiber $\pi^{-1}(\{x\})=\{x\} \times \mathcal{J} x$ is the $\mathcal{J}$-orbit of $x$ with distinguished root $x$. The action of the equivalence relation only changes this root and leaves the orbit invariant. Therefore, it acts by graph isomorphisms between the $\pi$-fibers. In this way, $G_{\mathcal{X}}$ is a graph over $\mathcal{R}(\mathcal{X})$.

A transversal function of $\mathcal{R}(\mathcal{X})$ is

$$
\nu: X \rightarrow \mathcal{M}(\mathcal{R}(\mathcal{X})), \nu^{x}:=\sum_{(x, y) \approx(x, x)} \delta_{(x, y)} .
$$

The measure $\mu$ on $X$ is invariant with respect to $\nu$. We let $M_{\mathcal{X}}=\mu \circ \eta$. The function $\mathfrak{u}_{0}:=1_{\{(x, x): x \in X\}}$ is an averaging function. By shifting with elements of $\mathcal{J}$ in the second variable and taking suitable linear combinations it can be made strictly positive. In this way, $\left(G_{\mathcal{X}}, M_{\mathcal{X}}\right)$ is a measure graph over $\mathcal{G}$.

Recall the definition of the random variable $\xi_{F}$ for a $\mathcal{R}(\mathcal{X})$-invariant function $F: V \rightarrow[0, \infty]$ (see Lemma 1.13). By Lemma 1.15 its integral satisfies

$$
\int \xi_{F}=\int_{V} F \mathfrak{u}_{0} \mathrm{~d} M_{\mathcal{X}}=\int_{X} F((x, x)) \mathrm{d} \mu .
$$

Remark 1.24. The considerations of this section also imply that the fractal graphs studied in 20] fall into our framework. Indeed from the considerations of [20] one can easily see that they give rise to a limit graphing. Hence, they can be treated by our methods. We refrain from giving details here.

\section{The Ihara Zeta function of a measure Graph}

In this section we discuss how a general measure graph over a groupoid gives immediately rise to a Zeta function. We will call this the Ihara Zeta function. For finite and for periodic graphs it will be shown to agree with the 'usual' Zeta function.

2.1. The definition and basic properties. Before introducing the Zeta function we need some further graph theoretic notions. Let a graph $G=(V, E)$ be given. A circle or closed path is a path $\left(e_{1}, \ldots, e_{n}\right)$ such that $t\left(e_{n}\right)=o\left(e_{1}\right)$.

Definition 2.1 (Reduced paths). Let $G=(V, E)$ be a graph.

(a) A closed path $\left(e_{1}, \ldots, e_{n}\right)$ has a backtrack if $e_{i+1}=\bar{e}_{i}$ for some $i \in\{1, \ldots, n-1\}$. A path with no backtracking is said to be proper.

(b) We call a circle primitive if it is not obtained by going $n \geq 2$ times around a shorter closed path.

(c) A closed path $\left(e_{1}, \ldots, e_{n}\right)$ has a tail if there is a number $k \in \mathbb{N}$ such that $\bar{e}_{j}=e_{n-j+1}$ for $1 \leq j \leq k$. 
(d) A closed path without backtracking or tail is called reduced.

Definition 2.2 (Counting of closed path). Whenever we are given a graph $G=(V, E)$, we let $N_{j}^{G}(x)$ be the number of reduced closed paths of length $j$ starting at a given vertex $x \in V$. Similarly, $P_{j}^{G}(x)$ will denote the number of primitive reduced circles of length $j$ starting at $x$. If the graph is understood from the context, we will suppress the superscript $G$ and just write $N_{j}(x)$ and $P_{j}(x)$.

Lemma 2.3 (Averaged circle counting). Let $\mathcal{G}$ be a groupoid and let $(G, M)$ be a measure graph over $\mathcal{G}$. Then, for any natural number $j$ the functions $\xi_{N_{j}}$ and $\xi_{P_{j}}$ given by

$$
\Omega \ni \omega \mapsto \xi_{N_{j}}^{\omega}:=\sum_{x \in \pi^{-1}(\omega)} N_{j}(x) \delta_{x} \in \mathcal{M}(V),
$$

and

$$
\Omega \ni \omega \mapsto \xi_{P_{j}}^{\omega}:=\sum_{x \in \pi^{-1}(\omega)} P_{j}(x) \delta_{x} \in \mathcal{M}(V)
$$

are random variables. In particular, the numbers

$$
\bar{N}_{j}:=\int \xi_{N_{j}}=\int_{\Omega} \xi_{N_{j}}^{\omega}(\mathfrak{u}) d m(\omega)=\int_{V} N_{j}(x) \mathfrak{u}(x) d M(x),
$$

and

$$
\bar{P}_{j}:=\int \xi_{P_{j}}=\int_{\Omega} \xi_{P_{j}}^{\omega}(\mathfrak{u}) d m(\omega)=\int_{V} P_{j}(x) \mathfrak{u}(x) d M(x)
$$

exist and do not depend on the choice of $\mathfrak{u}$, provided $\nu * \mathfrak{u}=1$.

Proof. The functions $N_{j}$ and $P_{j}$ only depend on the isomorphism class of a finite ball around the corresponding points. Hence, they are clearly measurable by the definition of a measurable graph. Moreover, they are invariant under the action of the groupoid as this action results in graph isomorphisms and hence preserves the isomorphism classes of finite balls around the corresponding points. Thus, by Proposition 1.13 the functions $\xi_{N_{j}}$ and $\xi_{P_{j}}$ are random variables. The remaining statements follow from the considerations concerning the integration of random variables in the previous section and specifically from Definition 1.16.

Definition 2.4 (The Zeta function of a measure graph). Let $(G, M)$ be a measure graph over a groupoid. The complex function

$$
Z(u):=Z_{(G, M)}(u):=\exp \left(\sum_{j \geq 1} \frac{\bar{N}_{j}}{j} u^{j}\right)
$$

is called Ihara Zeta function of $(G, M)$. The numbers $\bar{N}_{j}, j \in \mathbb{N}$, appearing in its definition are called the coefficients of the Zeta function.

Proposition 2.5. Let $(G, M)$ be a measure graph over a groupoid. $Z=Z_{(G, M)}$ is a holomorphic function on the disc $B_{(D-1)^{-1}}:=\left\{u \in \mathbb{C}:|u|<(D-1)^{-1}\right\}$, where $D$ is the maximal vertex degree of $G$. Furthermore, $Z$ has an Euler product representation, i.e., for each $u \in B_{(D-1)^{-1}}$ the equality

$$
Z(u)=\prod_{j \geq 1}\left(1-u^{j}\right)^{-\frac{\bar{P}_{j}}{j}}
$$

holds. 
Proof. The first assertion is a direct consequence of the observation $N_{j}(x) \leq D(D-1)^{j-1}$ and the finiteness of the measure $\mathfrak{u} \mathrm{d} M$. For the second statement let us note that for each $j$ and each $x \in V$ the equality

$$
N_{j}(x)=\sum_{i \mid j} P_{i}(x)
$$

holds, where $i \mid j$ means that $i$ divides $j$. Thus, using an integrated version of this identity we obtain

$$
\begin{aligned}
\sum_{j \geq 1} \frac{\bar{N}_{j}}{j} u^{j} & =\sum_{j \geq 1} \sum_{i \mid j} \bar{P}_{i} \frac{u^{j}}{j} \\
\text { (interchanging summation) } & =\sum_{i \geq 1} \sum_{j \geq 1} \bar{P}_{i} \frac{u^{i j}}{i j} \\
\text { (logarithmic series) } & =\sum_{i \geq 1}-\log \left(1-u^{i}\right) \frac{\bar{P}_{i}}{i} .
\end{aligned}
$$

Note that for $|u|<(D-1)^{-1}$ all the above series are absolutely convergent. Taking exponentials yields the claim.

Remark 2.6. We emphasize that the validity of the Euler product representation of $Z$ only owes to the validity of

$$
N_{j}(x)=\sum_{i \mid j} P_{i}(x)
$$

2.2. Examples. Here we discuss the Zeta functions of the measure graphs which were introduced in Subsection 1.4. It is quite instructive to compute the quantities $\bar{N}_{j}$ and $\bar{P}_{j}$ for these examples.

For finite and periodic graphs the exponent $\bar{P}_{j}$ in the Euler product representation can be further resolved and the product then be taken over certain geometric quantities. For this purpose we recall the following graph theoretic notions.

Definition 2.7 (Cycles).

(a) Two closed paths $\left(e_{1}, \ldots, e_{m}\right)$ and $\left(f_{1}, \ldots, f_{m}\right)$ are said to be equivalent if there is some $l$ such that $e_{i}=f_{i+l}$ for all $1 \leq i \leq m$. Here we use the convention $f_{i}=f_{i+m}$. The corresponding equivalence classes of closed paths are called cycles. The equivalence class of a closed path $C$ is denoted by $[C]$.

(b) Cycles with their representatives being reduced and primitive are called prime cycles. The set of all prime cycles is denoted by $\mathcal{P}$.

2.2.1. Finite graphs. Let $G=(V, E)$ be finite. As discussed at the end of the previous section $G$ is a measure graph over the trivial groupoid. The measure $M$ can be chosen to be the counting measure on $V$. Then, the constant function $1_{V}$ is an averaging function. In this situation we obtain

$$
\bar{N}_{j}=\sum_{x \in V} N_{j}(x) \text { and } \bar{P}_{j}=\sum_{x \in V} P_{j}(x),
$$

that is, $\bar{N}_{j}$ is the total number of reduced closed paths of length $j$ and $\bar{P}_{j}$ is the total number of primitive reduced closed paths of length $j$. 
Proposition 2.8. Let $G=(V, E)$ be a finite graph and let $M$ be the counting measure. Then the Zeta function satisfies

$$
Z_{(G, M)}(u)=\prod_{[P] \in \mathcal{P}}\left(1-u^{\ell(P)}\right)^{-1}
$$

where $\mathcal{P}$ is the set of prime cycles and $\ell(P)$ is the length of $[P]$.

Proof. Since $M$ is the counting measure on $V$ the quantity $\bar{P}_{j}$ coincides with the number of primitive reduced closed paths of length $j$. Thus, we have $\bar{P}_{j}=j \cdot\left|\mathcal{P}_{j}\right|$, with $\mathcal{P}_{j}$ being the set of prime cycles of length $j$. Using this fact and reordering the product of Proposition 2.5 yields the claim.

Remark 2.9. For a finite graph the Ihara Zeta function is 'usually' defined via the product of the previous proposition (see e.g. 35]). Thus, for finite graphs our definition of Zeta function coincides with the 'usual' one.

Our approach suggests to also consider the Zeta function coming from the normalized measure giving the mass $1 /|V|$ to any vertex. We call the arising Zeta function the normalized Zeta function of the finite graph and denote it by $Z_{G, \text { norm }}$. Obviously $Z_{G \text {, norm }}^{|V|}=Z_{(G, M)}$ holds.

2.2.2. Periodic graphs. Let us first fix some notation. For a group $\Upsilon$ acting on some space $X$ we let $\Upsilon x=\{\gamma x: \gamma \in \Upsilon\}$ be the orbit and $\Upsilon_{x}=\{\gamma \in \Upsilon: \gamma x=x\}$ be the stabilizer of $x \in X$. Further, we denote by $X / \Upsilon=\{\Upsilon x: x \in X\}$ the collection of orbits.

Let $(G, \Gamma)$ be a periodic graph (see Definition [1.20). Recall that in this case $M=m \circ \eta$ is the counting measure on the vertices and $(G, M)$ is a measure graph over the groupoid $\mathcal{G}=\Gamma$. In this situation we obtain

$$
\bar{N}_{j}=\sum_{x \in F} \frac{N_{j}(x)}{\left|\Gamma_{x}\right|} \text { and } \bar{P}_{j}=\sum_{x \in F} \frac{P_{j}(x)}{\left|\Gamma_{x}\right|} .
$$

As these quantities (and thus $Z_{(G, M)}$ ) only depend on $G$ and $\Gamma$ we shall write $Z_{(G, \Gamma)}$ for the corresponding zeta function.

The group $\Gamma$ naturally acts on closed paths and on cycles of $G$. Namely, for a closed path $C=\left(e_{1}, \ldots, e_{m}\right)$ and $\gamma \in \Gamma$ we let $\gamma C=\left(\gamma e_{1}, \ldots, \gamma e_{m}\right)$. This action is compatible with passing from closed paths to cycles. Thus, for a cycle $[C]$ we can set $\gamma[C]=[\gamma C]$.

Proposition 2.10. Let $(G, \Gamma)$ be a periodic graph. Its Zeta function satisfies

$$
Z_{(G, \Gamma)}(u)=\prod_{\Gamma[P] \in \mathcal{P} / \Gamma}\left(1-u^{\ell(P)}\right)^{-\frac{1}{\mid \Gamma_{[P]}}},
$$

where $\mathcal{P}$ is the set of all prime cycles and $\ell(P)$ is the length of $[P]$.

Proof. We first note that the cardinality of the stabilizer $\left|\Gamma_{[P]}\right|$ and the length $\ell(P)$ are independent of the chosen representative of $\Gamma[P]$. Now the statement follows from Proposition 2.5 by reordering the product once we show the equality

$$
\frac{\bar{P}_{j}}{j}=\sum_{\{\Gamma[P] \in \mathcal{P} / \Gamma: \ell(P)=j\}} \frac{1}{\left|\Gamma_{[P]}\right|} .
$$


For any prime cycle $[P] \in \mathcal{P}$ the group $\Gamma_{[P]}$ acts on the set $[P]$. Hence, for $Q \in[P]$ The orbit-stabilizer formula yields

$$
\left|\Gamma_{[P]}\right|=\left|\Gamma_{[P]} Q\right|\left|\Gamma_{Q}\right|,
$$

where we use that the stabilizer of $Q$ of the $\Gamma_{[P]}$ action is $\Gamma_{Q}$. If we denote the set of all primitive reduced closed paths by $\widetilde{\mathcal{P}}$, this leads to

$$
\begin{aligned}
\sum_{\{\Gamma P \in \widetilde{\mathcal{P}} / \Gamma: \ell(P)=j\}} \frac{1}{\left|\Gamma_{P}\right|} & =\sum_{\{\Gamma P \in \widetilde{\mathcal{P}} / \Gamma: \ell(P)=j\}} \frac{\left|\Gamma_{[P]} P\right|}{\left|\Gamma_{[P]}\right|} \\
& =\sum_{\{\Gamma[P] \in \mathcal{P} / \Gamma: \ell(P)=j\}} \sum_{\Gamma Q \in \Gamma[P]} \frac{\left|\Gamma_{[Q]} Q\right|}{\left|\Gamma_{[Q]}\right|} \\
& =\sum_{\{\Gamma[P] \in \mathcal{P} / \Gamma: \ell(P)=j\}} \frac{1}{\left|\Gamma_{[P]}\right|} \sum_{\Gamma Q \in \Gamma[P]}\left|\Gamma_{[Q]} Q\right| .
\end{aligned}
$$

Let now $\left\{P_{1}, \ldots, P_{l}\right\}$ be a collection of Elements of $[P]$ of minimal cardinality such that

$$
\left\{\Gamma P_{1}, \ldots, \Gamma P_{l}\right\}=\Gamma[P] .
$$

It is readily verified that the sets $\Gamma_{[P]} P_{1}, \ldots, \Gamma_{[P]} P_{l}$ are a partition of $[P]$. Since $|[P]|=\ell(P)=$ $j$ and $\left[P_{k}\right]=[P]$ for $1 \leq k \leq l$, we obtain

$$
\sum_{\Gamma Q \in \Gamma[P]}\left|\Gamma_{[Q]} Q\right|=\sum_{k=1}^{l}\left|\Gamma_{\left[P_{k}\right]} P_{k}\right|=\sum_{k=1}^{l}\left|\Gamma_{[P]} P_{k}\right|=j .
$$

Hence, in order to prove the desired identity for $\bar{P}_{j} / j$, it suffices to verify

$$
\bar{P}_{j}=\sum_{\{\Gamma P \in \widetilde{\mathcal{P}} / \Gamma: \ell(P)=j\}} \frac{1}{\left|\Gamma_{P}\right|} .
$$

To this end, fix a fundamental domain $F \subset V$ of finite co-volume. For each orbit $\Gamma P \in \widetilde{\mathcal{P}} / \Gamma$ there is a unique $x \in F$ such that there exist paths in $\Gamma P$ starting in $x$. Thus, we say $\Gamma P$ starts in $x \in F$ if one of the paths in $\Gamma P$ starts in $x$. We obtain

$$
\sum_{\{\Gamma P \in \widetilde{\mathcal{P}} / \Gamma: \ell(P)=j\}} \frac{1}{\left|\Gamma_{P}\right|}=\sum_{x \in F} \sum_{\substack{\{\Gamma P \in \mathcal{\mathcal { P }} / \Gamma: \ell(P)=j \\ \text { and } \Gamma P \text { starts in } x\}}} \frac{1}{\left|\Gamma_{P}\right|} .
$$

In order to sum over paths starting in $x \in F$ (as required to compute $\bar{P}_{j}$ ) instead of orbits of paths we need to count how many different paths in $\Gamma P$ actually do start in $x$. Now if $\Gamma P$ starts at $x$, then $\gamma \Gamma P$ starts in $x$ as well if and only if $\gamma \in \Gamma_{x}$. Hence, the number of distinct paths starting at $x$ is given by $\mid\{Q \in \Gamma P: Q$ starts in $x\}|=| \Gamma_{x} / \Gamma_{P}|=| \Gamma_{x}|/| \Gamma_{P} \mid$. These considerations yield

$$
\sum_{x \in F} \sum_{\substack{\{\Gamma P \mathcal{\mathcal { P }} / \Gamma: \ell(P)=j \\ \text { and } \Gamma P \text { starts in } x\}}} \frac{1}{\left|\Gamma_{P}\right|}=\sum_{x \in F} \sum_{\substack{\{\Gamma P \in \mathcal{P} / \Gamma: \ell(P)=j \\ \text { and } \Gamma P \text { starts in } x\}}} \frac{1}{\left|\Gamma_{P}\right|} \cdot \frac{\left|\Gamma_{x}\right|}{\left|\Gamma_{x}\right|}=\sum_{x \in F} \frac{P_{j}(x)}{\left|\Gamma_{x}\right|}=\bar{P}_{j} .
$$

This finishes the proof. 
Remark 2.11. - In 5 periodic graphs were the first class of examples of infinite graphs for which Zeta functions were defined with the above product representation serving as a definition. Thus, our concept of Zeta function of a periodic graph coincides with the one previously studied.

- In 4 Zeta functions of vertex transitive infinite $q$-regular graphs were introduced. If the action of the automorphism group is free these coincide with our Zeta function for periodic graphs. If the action is not free they differ by some exponent.

- The previous proposition can be seen as an analogue of Theorem 2.2 (iii) of [19] which states that

$$
\prod_{\Gamma[P] \in \mathcal{P} / \Gamma}\left(1-u^{\ell(P)}\right)^{-\frac{1}{\Gamma[P]}}=\exp \left(\sum_{j \geq 1} \frac{N_{j}^{\Gamma}}{j} u^{j}\right),
$$

for a sequence of numbers $N_{j}^{\Gamma}$ that depend on geometric quantities of the graph. The crucial new insight is that the coefficients $N_{j}^{\Gamma}$ equal $\bar{N}_{j}$ and can thus be expressed as integrals over functions on the vertices of the graph. Indeed, this is the main observation that lies at the heart of our paper.

2.2.3. Percolation graphs. Let $\left(G_{\Gamma, \text { perc }}, M_{\mathbb{P}}\right)$ be a percolation graph as in Section 1.4.3, We obtain

$$
\bar{N}_{j}=\int_{\Theta} N_{j}^{G_{\Gamma, \text { perc }}}((e, \theta)) \mathrm{d} \mathbb{P}=\int_{\Theta} N_{j}^{G_{\theta}}(e) \mathrm{d} \mathbb{P}
$$

and

$$
\bar{P}_{j}=\int_{\Theta} P_{j}^{G_{\Gamma, \text { perc }}}((e, \theta)) \mathrm{d} \mathbb{P}=\int_{\Theta} P_{j}^{G_{\theta}}(e) \mathrm{d} \mathbb{P} .
$$

This shows that the Zeta function of the percolation graph counts the averaged number of reduced closed paths which start at the neutral element in the realization $G_{\theta}$.

Remark 2.12. To the best of our knowledge Zeta functions of percolation graphs have not been discussed in the literature before. This underscores the generality of the approach proposed in the present paper.

2.2.4. Graphings. Let $\mathcal{X}$ be a graphing as in Subsection 1.4 .4 and let $\left(G_{\mathcal{X}}, M_{\mathcal{X}}\right)$ the corresponding measure graph. We obtain

$$
\bar{N}_{j}=\int_{X} N_{j}^{G \mathcal{X}}((x, x)) \mathrm{d} \mu \text { and } \bar{P}_{j}=\int_{X} P_{j}^{G \mathcal{X}}((x, x)) \mathrm{d} \mu .
$$

The graph $G_{x}=\left(\mathcal{J} x, E_{x}\right)$ with $E_{x}=\left\{(y, z) \in \mathcal{J} x \times \mathcal{J} x: y=\iota_{j} z\right.$ for some $\left.1 \leq j \leq n\right\}$ is isomorphic to the induced graph on the fiber $\pi^{-1}(\{x\}) \subseteq V_{\mathcal{X}}$. Therefore, the above formulas simplify to

$$
\bar{N}_{j}=\int_{X} N_{j}^{G_{x}}(x) \mathrm{d} \mu \text { and } \bar{P}_{j}=\int_{X} P_{j}^{G_{x}}(x) \mathrm{d} \mu .
$$

For the corresponding Zeta function we shall simply write $Z_{\mathcal{X}}$ instead of $Z_{\left(G_{\mathcal{X}}, M_{\mathcal{X}}\right)}$.

Remark 2.13. Zeta functions of graphings have occurred implicitly in the literature as the limit of Zeta functions of finite graphs. We will discuss this in detail in Subsection 5.1. 


\section{A determinant formula for the Zeta function}

In this section we provide a determinant formula for the Ihara Zeta function in terms of the adjacency and the degree operator. The determinant formula states that in a small neighborhood around 0 the reciprocal of the function $Z_{(G, M)}$ can be expressed as a determinant (up to some multiplicative factor). In particular, $Z_{(G, M)}$ must then be a non-vanishing holomorphic function in a small neighborhood of 0 . For the proof we will follow the lines of 20] and give details only when they need to be adapted to our situation. For the case of finite graphs the presented approach towards proving the determinant formula was first established in [34]. However, before providing the determinant formula we need to set the stage and introduce the operators and the determinant. To this end, we discuss the von Neumann algebra $\mathcal{N}(\mathcal{G}, G)$ of a measure graph and introduce a trace on it.

3.1. The von Neumann algebra. Let a measure graph $(G, M)$ over a groupoid $\mathcal{G}$ be given. Then, the graph can be seen as a bundle over $\Omega=\mathcal{G}^{(0)}$ via $\pi: V \rightarrow \Omega$. This induces a bundle structure on $L^{2}(G, M)$ and this will be our point of view in the subsequent considerations. More specifically, by Fubini's Theorem we can associate with $f \in L^{2}(V, m \circ \eta)$ a family $\left(f_{\omega}\right)_{\omega \in \Omega}$ of functions

for $m$-almost all $\omega \in \Omega$.

$$
f_{\omega} \in \ell^{2}\left(V^{\omega}, \eta^{\omega}\right)=\ell^{2}\left(V^{\omega}\right) \text { such that } f(x)=f_{\pi(x)}(x)
$$

Remark 3.1. On the technical level, our approach can be expressed via direct integral theory and the fact that there is a canonical isomorphism

$$
L^{2}(V, M) \simeq \int_{\Omega}^{\oplus} \ell^{2}\left(V^{\omega}, \eta^{\omega}\right) d m(\omega) .
$$

Here, the field $\omega \mapsto \ell^{2}\left(V^{\omega}, \eta^{\omega}\right)$ of Hilbert spaces is equipped with the canonical measurable structure induced by all measurable $f: \mathcal{G} \longrightarrow[0, \infty]$ with $\eta^{\omega}\left(f^{2}\right)<\infty$ for all $\omega \in \Omega$. In our presentation we will not need to explicitly invoke direct integral theory and for this reason we do not elaborate further on this. Instead we just recall (or rather adapt) the corresponding considerations of Section 3 of 23 to our context. The work [23] in turn is a specialization of corresponding considerations in [8]. We refer to these works for further discussion of the background and the technicalities. We also refer the reader to e.g. [11] for background in direct integral theory.

A special role will be played by those operators which respect the bundle structure of $L^{2}(V, M)$ given by (3.1). Namely, we say that the (not necessarily bounded) operator $A$ : $L^{2}(V, M) \rightarrow L^{2}(V, M)$ is decomposable if there exist operators $A_{\omega}: \ell^{2}\left(V^{\omega}, \eta^{\omega}\right) \rightarrow \ell^{2}\left(V^{\omega}, \eta^{\omega}\right)$ such that $(A f)_{\omega}(x)=\left(A_{\omega} f_{\omega}\right)(x)$, for almost every $\omega \in \Omega$. We then write $A=\int_{\Omega}^{\oplus} A_{\omega} d m(\omega)$.

For $g \in \mathcal{G}$, let the unitary operator $U_{g}$ be given by

$$
U_{g}: \ell^{2}\left(V^{s(g)}, \eta^{s(g)}\right) \rightarrow \ell^{2}\left(V^{r(g)}, \eta^{r(g)}\right), U_{g} f(x):=f\left(g^{-1} x\right) .
$$

A family $\left(A_{\omega}\right)_{\omega \in \Omega}$ of bounded operators $A_{\omega}: \ell^{2}\left(V^{\omega}, \eta^{\omega}\right) \rightarrow \ell^{2}\left(V^{\omega}, \eta^{\omega}\right)$ is called a bounded random operator if it satisfies :

- $\omega \mapsto\left\langle g_{\omega}, A_{\omega} f_{\omega}\right\rangle$ is measurable for arbitrary $f, g \in L^{2}(V, M)$.

- There exists a $C \geq 0$ with $\left\|A_{\omega}\right\| \leq C$ for almost all $\omega \in \Omega$.

- The equivariance condition $A_{r(g)}=U_{g} A_{s(g)} U_{g}^{*}$ is satisfied for all $g \in \mathcal{G}$. 
Two bounded random operators $\left(A_{\omega}\right),\left(B_{\omega}\right)$ are called equivalent, $\left(A_{\omega}\right) \sim\left(B_{\omega}\right)$ if $A_{\omega}=B_{\omega}$ for $m$-almost every $\omega \in \Omega$. Each equivalence class of bounded random operators $\left(A_{\omega}\right)$ gives rise to a bounded operator $A$ on $L^{2}(V, M)$ by $A f(x):=A_{\pi(x)} f_{\pi(x)}$. This allows us to identify the class of $\left(A_{\omega}\right)$ with the bounded operator $A$. The set of classes of bounded random operators is denoted by $\mathcal{N}(\mathcal{G}, G)$. It is obviously an algebra (under the usual pointwise addition and multiplication on the level of representatives). Moreover, it carries a norm given by

$$
\left\|\left[\left(A_{\omega}\right)_{\omega \in \Omega}\right]\right\|:=\inf \left\{C \geq 0:\left\|A_{\omega}\right\| \leq C \text { for } m \text {-almost every } \omega \in \Omega\right\} .
$$

(It is not hard to see that this is indeed well-defined and a norm.) The following is the main theorem on the structure of the space of random operators (see [ 8 , Thm. V.2])

Theorem 3.2. The set $\mathcal{N}(\mathcal{G}, G)$ of classes of bounded random operators is a von Neumann algebra.

3.1.1. The canonical trace. The admissible setting of 23 always gives a canonical weight on the von Neumann algebra in question. In this section we present details for our situation and show, in particular, that then this weight is actually a trace. Further details can be found in Section 4 of 23 .

Let a measure graph $(G, M)$ over a groupoid $\mathcal{G}$ be given and $\mathcal{N}(\mathcal{G}, G)$ be the associated von Neumann algebra. Let $\mathcal{N}^{+}(\mathcal{G}, G)$ denote the set of non-negative self-adjoint operators in $\mathcal{N}(\mathcal{G}, G)$. We will show that every operator $A \in \mathcal{N}^{+}(\mathcal{G}, G)$ gives rise to a new random variable $\beta_{A}$. Integrating this random variable, we obtain a weight on $\mathcal{N}(\mathcal{G}, G)$. This weight will be shown to be a trace. We start by associating a transversal function as well as a random variable with each element in $\mathcal{N}^{+}(\mathcal{G}, G)$. For a nonnegative function $f$ on $\mathcal{G}^{\omega}$ we will denote by $q_{\omega}(f)$ the function on $\pi^{-1}(\omega)$ given by

$$
q_{\omega}(f)(x)=\int_{\mathcal{G}^{\omega}} \mathfrak{u}\left(g^{-1} x\right) f(g) d \nu^{\omega}(g)
$$

for all $x \in V$. Thus, $q_{\omega}(f)$ is the restriction of $q(f)$ as defined at the end of Section 1.2 to the fiber $V^{\omega}$.

To proceed we will need the (usual) trace tr on the set of non-negative operators on an Hilbert space. This trace is defined by

$$
\operatorname{tr}(A):=\sum_{\iota \in I}\left\langle A e_{\iota}, e_{\iota}\right\rangle
$$

where $e_{\iota}, \iota \in I$, is an orthonormal basis. It does not depend on the choice of the orthonormal basis. In particular, is is invariant under conjugation with unitaries i.e. $\operatorname{tr}\left(U A U^{*}\right)=\operatorname{tr}(A)$ for any unitary $U$. Note that this trace may well take the value $\infty$. We will need this trace for the Hilbert space $\ell^{2}\left(V^{\omega}, \eta^{\omega}\right)$ for each $\omega \in \Omega$. Suppressing the dependence on $\omega \in \Omega$, we will still use the notation tr. For a function $f$ on $V^{\omega}$ we denote by $M_{f}$ the operator of multiplication by $f$ in $\ell^{2}\left(V^{\omega}, \eta^{\omega}\right)$. Similarly, for a function $f \in \mathcal{F}^{+}(V)$ we denote by $M_{f_{\omega}}$ the operator of multiplication by the restriction of $f$ to $V_{\omega}$.

We will need to take the trace of operators of the form $B M_{f} B$ for nonnegative measurable functions $f$ and bounded nonnegative operators $B$. To do so we note that - under the additional assumption of boundedness of $f$ - the operator $C:=M_{\sqrt{f}} B$ is bounded with adjoint given by $C^{*}=B M_{\sqrt{f}}$. Hence, we have for such $f$ that $B M_{f} B=C^{*} C$ is nonnegative and we can take the trace. For general nonnegative $f$ we define

$$
\operatorname{tr}\left(B M_{f} B\right):=\lim _{n \rightarrow \infty} \operatorname{tr}\left(B M_{f_{n}} B\right)
$$


with $f_{n}:=\max \{f, n\}$.

Lemma 3.3. Let $A \in \mathcal{N}^{+}(\mathcal{G}, G)$ be given.

(a) Then $\varphi_{A}$, given by $\varphi_{A}^{\omega}(f):=\operatorname{tr}\left(A_{\omega}^{1 / 2} M_{q_{\omega}(f)} A_{\omega}^{1 / 2}\right), f \in \mathcal{F}^{+}(\mathcal{G})$, defines a transversal function.

(b) Then $\xi_{A}$, given by $\xi_{A}^{\omega}(f):=\operatorname{tr}\left(A_{\omega}^{1 / 2} M_{f, \omega} A_{\omega}^{1 / 2}\right), f \in \mathcal{F}^{+}(V)$, is a random variable.

Proof. These statements are contained in Lemma 4.2 of [23]. For convenience of the reader we indicate the proof of (b). The proof of (a) can be done along very similar lines: Clearly the map $f \mapsto \xi_{A}(f)$ is monotone (i.e. $\xi_{A}(g) \leq \xi_{A}(f)$ for $\left.g \leq f\right)$. Hence, it is a measure. Moreover, this measure is supported on $V^{\omega}$ by the very definition of $M_{f, \omega}$. The invariance of $\xi_{A}$ follows now easily from the invariance properties of the $\left(A_{\omega}\right)$ and the invariance of the trace under conjugation with a unitary operator.

Let us recall the following definitions. A weight on a von Neumann algebra $\mathcal{N}$ is a map $\tau: \mathcal{N}^{+} \rightarrow[0, \infty]$ satisfying $\tau(A+B)=\tau(A)+\tau(B)$ and $\tau(\lambda A)=\lambda \tau(A)$ for arbitrary $A, B \in \mathcal{N}^{+}$and $\lambda \geq 0$. The weight is called normal if $\tau\left(A_{n}\right)$ converges to $\tau(A)$ whenever $A_{n}$ is an increasing sequence of operators (i.e. $A_{n} \leq A_{n+1}, n \in \mathbb{N}$ ) converging strongly to $A$. It is called faithful if $\tau(A)=0$ implies $A=0$. It is called semifinite if $\tau(A)=\sup \{\tau(B): B \leq$ $A, \tau(B)<\infty$ \}. If a weight $\tau$ satisfies $\tau\left(C C^{*}\right)=\tau\left(C^{*} C\right.$ ) for arbitrary $C \in \mathcal{N}$ (or equivalently $\tau\left(U A U^{*}\right)=\tau(A)$ for arbitrary $A \in \mathcal{N}^{+}$and unitary $U \in \mathcal{N}$, cf. [11, Cor. 1 in I.6.1]), it is called a trace.

In our situation we have a canonical candidate for a weight at our disposal. This is introduced in the next proposition.

Proposition 3.4 (Introducing $\tau$ ). Let $(G, M)$ be a measure graph over $\mathcal{G}$. For $A \in \mathcal{N}^{+}(\mathcal{G}, G)$, the expression

$$
\tau(A):=\int_{\Omega} \operatorname{tr}\left(A_{\omega}^{\frac{1}{2}} M_{\mathfrak{u}_{\omega}} A_{\omega}^{\frac{1}{2}}\right) d m(\omega) \in[0, \infty]
$$

does not depend on $\mathfrak{u} \in \mathcal{F}^{+}(G)$ provided $\nu * \mathfrak{u} \equiv 1$.

Proof. This follows directly from Lemma 1.15 and Lemma 3.3 .

We are going to show that the map

$$
\tau: \mathcal{N}^{+}(\mathcal{G}, G) \rightarrow[0, \infty], A \mapsto \tau(A),
$$

is a faithful, semifinite normal trace on $\mathcal{N}(\mathcal{G}, G)$. In order to do so we will present some additional pieces of information. These may also be interesting in their own right.

An operator $K$ on $L^{2}(V, M)$ is called a Carleman operator (cf. [36] for further details) if there exists a $k \in \mathcal{F}\left(V^{(2)}\right)$ with

$$
k(x, \cdot) \in \ell^{2}\left(V^{\pi(x)}, \eta^{\pi(x)}\right) \text { for all } x \in V
$$

such that for any $f \in L^{2}(V, M)$

$$
K f(x)=\int_{V^{\pi(x)}} k(x, y) f(y) d \eta^{\pi(x)}(y)=\sum_{y \in V^{\pi(x)}} k(x, y) f(y)=: K_{\pi(x)} f_{\pi(x)}(x)
$$

in the sense of $L^{2}(V, M)$. This $k$ is called the kernel of $K$ : Obviously, $K=\int_{\Omega}^{\oplus} K_{\omega} d m$. Let $\mathcal{K}$ be the set of all Carleman operators satisfying for all $g \in \mathcal{G}$

$$
k(g x, g y)=k(x, y)
$$


for all $x, y \in V^{(2)}$.

For a Carleman operator $K$ the expressions $\tau\left(K K^{*}\right)$ and $\tau\left(K^{*} K\right)$ can directly be calculated.

Proposition 3.5 (Proposition 4.5 of [23]). Let $(G, M)$ be a measure graph over $\mathcal{G}$. Let $K \in \mathcal{K}$ with kernel $k$ be given. Then we have

$$
\tau\left(K^{*} K\right)=\int_{\Omega} \int_{V^{\omega}} \int_{V^{\omega}} \mathfrak{u}(y)|k(x, y)|^{2} d \eta^{\omega}(x) d \eta^{\omega}(y) d m(\omega)=\tau\left(K K^{*}\right),
$$

for any $\mathfrak{u} \in \mathcal{F}^{+}(V)$ satisfying $\nu * \mathfrak{u} \equiv 1$.

In our situation all elements of the von Neumann algebra are Carleman operators.

Proposition 3.6 $(\mathcal{N}(\mathcal{G}, G)=\mathcal{K})$. Let $(G, M)$ be a graph over $\mathcal{G}$. Then, any element of $\mathcal{N}(\mathcal{G}, G)$ is a Carleman operator.

Proof. As shown in Proposition 4.4 of 23 the set $\mathcal{K}$ is a right ideal in $\mathcal{N}(\mathcal{G}, G)$. Now, obviously, the identity is a Carleman operator (with kernel given by $k(x, y)=1$ if $x=y$ and $k(x, y)=0$ otherwise). Hence, the desired statement follows.

After these preparations we can now state (and prove) the main result of this subsection.

Theorem 3.7. Let $(G, M)$ be a measure graph over $\mathcal{G}$. Then, the map

$$
\tau: \mathcal{N}^{+}(\mathcal{G}, G) \rightarrow[0, \infty]
$$

is a faithful, semifinite, normal trace. Furthermore, this trace is finite, i.e., $\tau$ gives a finite value when applied to the identity. Thus, $\tau$ can be uniquely extended to a continuous linear map on all of $\mathcal{N}(\mathcal{G}, G)$.

Proof. It follows from 8 ] (see Theorem 4.2 of [23] as well) that $\tau$ is a faithful, semifinite weight. From the preceding two propositions it follows immediately that $\tau$ is a trace. Now, a direct calculation shows that for the identity $I$ we obtain

$$
\tau(I)=\int_{V} \mathfrak{u}(x) d M(x) .
$$

By definition of a measure graph the above integral is finite and the identity has a finite trace. Whenever $A$ is now a nonnegative element in the von Neuman algebra we have $A \leq\|A\| I$ (where $\|A\|$ denotes the norm of $A$ ). Thus, monotonicity of the trace gives

$$
0 \leq \tau(A) \leq\|A\| \tau(I) .
$$

So, the trace is a continuous functional on the nonnegative elements. Given this, existence and uniqueness of a continuous extension to the whole von Neuman algebra is straightforward.

We finish this section by introducing two special Carleman operators.

Proposition 3.8 (The adjacency and the degree operator). Let $a_{G}$ be the adjacency matrix of the graph $G$ and $\operatorname{deg}$ its degree function. Then $a_{G}$ is the kernel of a Carleman operator which belongs to $\mathcal{N}(\mathcal{G}, G)$. The associated operator will be denoted by $A_{G}$ and called the adjacency operator. Furthermore, multiplication by deg provides a Carleman operator belonging to $\mathcal{N}(\mathcal{G}, G)$ which will be denoted by $\operatorname{Deg}_{G}$ and called the degree operator. 
Proof. We first show the measurability statements of the corresponding kernels. The function $a_{G}$ is measurable on $V^{(2)}$ by definition of a measurable graph. The kernel of $\operatorname{Deg}_{G}$ is given by

$$
V^{(2)} \rightarrow \mathbb{R},(x, y) \mapsto 1_{\{(z, z): z \in V\}}(x, y) \operatorname{deg}(y) .
$$

The measurability of this function follows from the Propositions 1.4 and 1.5 and the definition of the $\sigma$-algebra on $V^{(2)}$. The invariance of both kernels under the action of $\mathcal{G}$ is clear as $\mathcal{G}$ acts upon $V$ by graph isomorphisms.

3.2. The determinant formula. In order to state the main theorem of this section we need a holomorphic determinant on certain operators of $\mathcal{N}(\mathcal{G}, G)$. A natural way to obtain such a functional is to set

$$
\operatorname{det}_{\tau}(T):=\exp \circ \tau \circ \log (T) .
$$

Here, the logarithm of an operator $T$ with $\|I-T\|<1$ is defined via the power series expansion of the main branch of the logarithm around 1. Namely, we let

$$
\log (T)=-\sum_{k=1}^{\infty} \frac{1}{k}(I-T)^{k} .
$$

Since the trace $\tau$ is continuous in the norm topology the determinant function

$$
\operatorname{det}_{\tau}:\{T \in \mathcal{N}(\mathcal{G}, G):\|T-I\|<1\} \rightarrow \mathbb{C}, T \mapsto \operatorname{det}_{\tau}(T)
$$

is holomorphic.

Remark 3.9. The above definition of a determinant functional suffices for the purposes of this paper. As discussed in [19] one can use holomorphic functional calculus to extend the definition of the determinant to operators whose convex hull of the spectrum does not contain 0 .

Definition 3.10 ( $L^{2}$-Euler characteristic). Let $(G, M)$ be a measure graph over a groupoid. We set $Q_{G}=\operatorname{Deg}_{G}-I$. The number

$$
\chi_{(G, M)}=\frac{1}{2} \tau\left(I-Q_{G}\right)
$$

is called the $L^{2}$-Euler characteristic of $(G, M)$.

Theorem 3.11 (Determinant formula). Let $(G, M)$ be a measure graph over a groupoid. Then, for $|u|<R^{-1}$ the Zeta function satisfies the equation

$$
Z_{(G, M)}(u)^{-1}=\left(1-u^{2}\right)^{-\chi(G, M)} \operatorname{det}_{\tau}\left(I-u A_{G}+u^{2} Q_{G}\right),
$$

where $R:=\frac{D+\sqrt{D^{2}+4 D}}{2}$ and $D$ is the maximal vertex degree of $G$.

The rest of this section is devoted to proving the theorem. As mentioned earlier, we closely follow [20] and omit some calculations that only require algebraic manipulations of identities of bounded operators.

We define a series of Carleman operators $A_{j}$ recursively via $A_{0}:=I, A_{1}:=A_{G}, A_{2}:=$ $A_{G}^{2}-Q_{G}-I$ and $A_{j}:=A_{j-1} A_{G}-A_{j-2} Q_{G}$, for $j \geq 3$. Let $a_{j}$ denote the corresponding kernels. The following lemma explains the importance of the $A_{j}$ 's. 
Lemma 3.12. For each $p, q \in V$ the kernel entry $a_{j}(p, q)$ is equal to the number of proper paths of length $j$ starting in $p$ and ending in $q$. Furthermore, for each $j$ the norms of the corresponding operators satisfy $\left\|A_{j}\right\| \leq R^{j}$, where $R:=\frac{D+\sqrt{D^{2}+4 D}}{2}$ and $D$ is the maximal vertex degree of $G$.

Proof. Note that multiplying Carleman operators is essentially done via matrix multiplication with the corresponding kernels. Thus, the first statement can be deduced as in the proof of Lemma 5.2 in [20]. For the statement on the norm we note that the operator $A_{G}$ is given by the family $\left(A_{G, \omega}\right)$, where $A_{G, \omega}$ is the adjacency operator on the fiber $V^{\omega}$. Thus, for $f=\left(f_{\omega}\right) \in L^{2}(V, M)$ we obtain

$$
\left\|A_{G} f\right\|^{2}=\int_{\Omega}\left\|A_{G, \omega} f_{\omega}\right\|^{2} d m(\omega) \leq D^{2} \int_{\Omega}\left\|f_{\omega}\right\|^{2} d m(\omega)=D^{2}\|f\|^{2},
$$

showing $\left\|A_{1}\right\|=\left\|A_{G}\right\| \leq D$. As $Q_{G}+I$ is multiplication by deg we obtain $\left\|A_{2}\right\| \leq D^{2}+D$. The rest follows by induction on $j$, using the inequality $\left\|A_{j}\right\| \leq D\left(\left\|A_{j-1}\right\|+\left\|A_{j-2}\right\|\right)$.

The functions $a_{j}$ count the number of proper paths. As such paths may still have tails we need to introduce some further quantity. Following the notation in [20] we let $t_{j}(x)$ be the number of proper closed paths of length $j$ starting in $x$ which have a tail. By the definition of a measure graph the function $V \ni x \mapsto t_{j}(x)$ is measurable and we set

$$
t_{j}:=\int_{V} t_{j}(x) \mathfrak{u}(x) d M(x) .
$$

Lemma 3.13. (a) For each $j \geq 0$ we have $\bar{N}_{j}=\tau\left(A_{j}\right)-t_{j}$.

(b) $t_{1}=t_{2}=0$, and, for $j \geq 3, t_{j}=t_{j-2}+\tau\left(\left(Q_{G}-I\right) A_{j-2}\right)$.

(c) For each $j \geq 0$ the equality $t_{j}=\tau\left(\left(Q_{G}-I\right) \sum_{i=1}^{\left[\frac{j-1}{2}\right]} A_{j-2 i}\right)$ holds.

Proof. By Lemma 3.12 we have $N_{j}(x)=a_{j}(x, x)-t_{j}(x)$. Integrating with respect to the measure $\mathfrak{u} d M$ yields statement (a). Next we turn to proving statement (b). Whenever $(x, y)$ is an edge we let $\widetilde{t}_{j}(x, y)$ be the number of reduced closed paths of length $j$ with tail starting with the edge $(x, y)$. If $(x, y)$ is not an edge we set $\widetilde{t}_{j}(x, y)=0$. The function $\widetilde{t}_{j}$ is the kernel of an operator belonging to $\mathcal{N}(\mathcal{G}, G)$. Indeed, the required measurability of $\widetilde{t}_{j}$ follows from Proposition 1.4 (c) and the invariance under the action of the groupoid is clear, as it acts by graph isomorphisms. We obtain

$$
\begin{aligned}
\int_{V} t_{j}(x) \mathfrak{u}(x) d M(x) & =\int_{V} \mathfrak{u}(x) \sum_{\{y: y \sim x\}} \widetilde{t}_{j}(x, y) d M(x) \\
& =\int_{\Omega} \sum_{x \in V^{\omega}} \sum_{y \in V^{\omega}} \mathfrak{u}(x) \widetilde{t}_{j}(x, y) d m(\omega) \\
& =\int_{\Omega} \sum_{y \in V^{\omega}} \sum_{x \in V^{\omega}} \mathfrak{u}(y) \widetilde{t}_{j}(x, y) d m(\omega) \\
& =\int_{V} \mathfrak{u}(y) \sum_{\{x: x \sim y\}} \widetilde{t}_{j}(x, y) d M(y),
\end{aligned}
$$

where the third equality is due to the invariance of $\widetilde{t}_{j}(x, y)$ under the action of the groupoid and the invariance of $m$ (use Proposition 3.5 with the kernel $k(x, y)=\widetilde{t}_{j}(x, y)^{1 / 2}$ ). Now (b) 
can be obtained from the equation

$$
\sum_{\{x: x \sim y\}} \widetilde{t}_{j}(x, y)=t_{j-2}(y)+(\operatorname{deg}(y)-2) a_{j-2}(y, y),
$$

which is a consequence of the following observation: Each closed of length $j$ with tail can be shortened to a closed path of length $j-2$ by removing its first and last edge. Thus, we have to count the number of ways in which one can extend reduced closed paths of length $j-2$ starting in $y$ to reduced closed paths with tail starting in neighbors of $y$. There are two possibilities: Either such a path of length $j-2$ has a tail or not. There are exactly $t_{j-2}(y)$ reduced closed paths with tail starting in $y$. If one wants to avoid backtracking, each of these can be extended in $\operatorname{deg}(y)-1$ ways to neighbors of $y$. There are $a_{j-2}(y, y)-t_{j-2}(y)$ reduced closed paths without tails. To avoid backtracking there are $\operatorname{deg}(y)-2$ possibilities to extend each of them. Thus, we obtain

$$
\sum_{\{x: x \sim y\}} \widetilde{t}_{j}(x, y)=(\operatorname{deg}(y)-1) t_{j-2}(y)+(\operatorname{deg}(y)-2)\left(a_{j-2}(y, y)-t_{j-2}(y)\right)
$$

and the claim follows after integrating these identities. Assertion (c) is an immediate consequence of (b).

Lemma 3.14. For $j \geq 0$ let $B_{j}:=A_{j}-\left(Q_{G}-I\right) \sum_{i=1}^{[j / 2]} A_{j-2 i}$. Then

(a)

(b)

$$
\tau\left(B_{j}\right)= \begin{cases}\bar{N}_{j}-\tau\left(Q_{G}-I\right), & j \text { even } \\ \bar{N}_{j}, & j \text { odd }\end{cases}
$$

$$
\tau\left(\sum_{j \geq 1} B_{j} u^{j}\right)=\tau\left(-u \frac{d}{d u} \log \left(I-A_{G} u+Q_{G} u^{2}\right)\right),|u|<R^{-1} .
$$

Proof. The algebraic manipulations required to prove (a) are contained in the proof of Lemma 7.2 of [20]. To carry them out in our setting one only needs the identities of Lemma 3.13. Assertion (b) is given by Corollary 7.4 of [20] whose proof involves algebraic manipulations of power series that require the estimate on the norm of the $A_{j}$ of Lemma 3.12,

Proof of Theorem 3.11. Since $\tau$ is norm continuous we obtain by Lemma 3.14 (a) that the equality

$$
\begin{aligned}
\tau\left(\sum_{j \geq 1} B_{j} u^{j}\right) & =\sum_{j \geq 1} \tau\left(B_{j}\right) u^{j}=\sum_{j \geq 1} \bar{N}_{j} u^{j}-\sum_{j \geq 1} \tau\left(Q_{G}-I\right) u^{2 j} \\
& =\sum_{j \geq 1} \bar{N}_{j} u^{j}-\tau\left(Q_{G}-I\right) \frac{u^{2}}{1-u^{2}}
\end{aligned}
$$

holds. This computation together with 3.14 (b) yields

$$
\begin{aligned}
& u \frac{d}{d u} \log Z_{(G, M)}=\sum_{j \geq 1} \bar{N}_{j} u^{j} \\
& \quad=\tau\left(-u \frac{d}{d u} \log \left(I-A_{G} u+Q_{G} u^{2}\right)\right)-\frac{u}{2} \frac{d}{d u} \log \left(1-u^{2}\right) \tau\left(Q_{G}-I\right) .
\end{aligned}
$$


Dividing by $u$ for $u \neq 0$, integrating from 0 to $u$ and taking exponentials yield the claim.

\section{ZETA FUNCTION AND INTEGRATED DENSITY OF STATES ON ESSENTIALly REGULAR GRAPHS}

In this section we discuss properties of the Ihara Zeta function for essentially regular graphs. As for regular graphs in the finite case, their Zeta function is closely related to the spectrum of the adjacency operator.

Definition 4.1 (Essentially regular graph). Let $(G, M)$ be a measure graph over a groupoid. Then, $G$ is called essentially $(r+1)$-regular if $\operatorname{deg}(x)=r+1$ for $M$-almost all $x \in V$.

Let $(G, M)$ be a measure graph over a groupoid $\mathcal{G}$. Recall that each self-adjoint operator $T \in \mathcal{N}(\mathcal{G}, G)$ possesses a spectral measure $\mu_{T}$ on the spectrum $\sigma(T)$ of $T$ which is uniquely determined by the identity

$$
\tau(\varphi(T))=\int_{\sigma(T)} \varphi(\lambda) d \mu_{T}(\lambda), \text { for each } \varphi \in C(\sigma(T)) .
$$

Remark 4.2. The measure $\mu_{T}$ is sometimes called the abstract integrated density of states of $T$, see Chapters 5 and 6 of 23 ] for a detailed discussion.

We let $\mu_{G}:=\mu_{A_{G}}$ be the spectral measure of the adjacency operator of $(G, M)$. The following is the main observation of this paragraph. It connects the spectral theory of $A_{G}$ and the Zeta function $Z_{(G, M)}$.

Theorem 4.3. Let $(G, M)$ be a measure graph over a groupoid $\mathcal{G}$. Assume that $(G, M)$ is essentially $(r+1)$-regular. Then, for $|u|<R^{-1}$ its Zeta function satisfies

$$
Z_{(G, M)}(u)^{-1}=\left(1-u^{2}\right)^{\frac{(r-1) \tau(I)}{2}} \exp \left(\int_{\sigma\left(A_{G}\right)} \log \left(1-u \lambda+u^{2} r\right) d \mu_{G}(\lambda)\right),
$$

where $2 R=(r+1)+\sqrt{(r+1)^{2}+4(r+1)}$ and $\log$ is the main branch of the complex logarithm on the sliced plane $\mathbb{C}_{-}:=\mathbb{C} \backslash\{x \in \mathbb{R}: x \leq 0\}$.

Proof. To prove the theorem we will compute the quantities involved in the determinant formula of Theorem 3.11. As $G$ is essentially $(r+1)$-regular, the operator $Q_{G}=\operatorname{Deg}_{G}-I$ is multiplication by the constant $r$. Thus, we obtain

$$
\chi_{(G, M)}=\frac{1}{2} \tau\left(I-Q_{G}\right)=\frac{(1-r) \tau(I)}{2} .
$$

Using the inclusion $\sigma\left(A_{G}\right) \subseteq[-(r+1), r+1]$ and $r<R$ an elementary computation shows $1-\lambda u+u^{2} r \in \mathbb{C}_{-}$, whenever $|u|<R^{-1}$ and $\lambda \in \sigma\left(A_{G}\right)$. Thus, for all $|u|<R^{-1}$ the function $\lambda \mapsto \psi(\lambda):=\log \left(1-u \lambda+r u^{2}\right)$ is continuous on $\sigma\left(A_{G}\right)$. We obtain

$$
\log \left(1-u A_{G}+u^{2} Q_{G}\right)=\log \left(1-u A_{G}+u^{2} r I\right)=\psi\left(A_{G}\right) .
$$

Hence, the definition of $\mu_{G}$ yields

$$
\tau\left(\log \left(1-u A_{G}+u^{2} Q_{G}\right)\right)=\int_{\sigma\left(A_{G}\right)} \psi(\lambda) d \mu_{G}(\lambda) .
$$

Now the claim follows from Theorem 3.11 
Remark 4.4. In [16] the previous theorem serves as a definition for the Ihara Zeta function of certain infinite regular graphs. There, the measure $\mu_{G}$ is given by the integrated density of states (the KNS-spectral measure) of a weakly convergent graph sequence. Furthermore, for vertex-transitive (regular) graphs the above formula recovers Theorem 1.3 of 4 which was shown there by different means via an analysis of Bessel functions and heat kernels. In both cases the authors consider spectral measures associated with the Laplacian $L=\operatorname{Deg}_{G}-A_{G}$ instead of measures corresponding to $A_{G}$. However, for essentially regular graphs the operator $\operatorname{Deg}_{G}$ is a constant multiple of the identity and, thus, the Laplacian and the adjacency matrix are essentially the same operators up to a shift by a constant.

Corollary 4.5. Let $(G, M)$ be a measure graph over a groupoid $\mathcal{G}$. Assume that $(G, M)$ is essentially $(r+1)$-regular. Then its Zeta function can be continued to a holomorphic function on the open set

$$
\mathcal{O}:=\left\{z \in \mathbb{C}:|z|<r^{-1 / 2}\right\} \backslash\left\{x \in \mathbb{R}: r^{-1} \leq|x| \leq 1\right\} .
$$

Proof. Theorem 4.3, the inclusion $\sigma\left(A_{G}\right) \subseteq[-(r+1), r+1]$ and the elementary fact that $1-\lambda u+u^{2} r \in \mathbb{C}_{-}$for each pair $(u, \lambda) \in \mathcal{O} \times[-(r+1), r+1]$ show the claim.

\section{Convergence of measure graphs and of Zeta functions}

In this section we provide a general result on convergence of Zeta functions. More specifically, we introduce the notion of weak convergence of measure graphs and show that it implies local compact convergence of their Zeta functions. Our notion of weak convergence of measure graphs generalizes the concept of weak convergence of sequences of finite graphs which has been introduced in [3. While not noted explicitly, particular cases of weak convergence of finite graphs are at the core of all the earlier attempts to provide a Zeta function for infinite graphs via approximation [6, 16, 19, 20]. Therefore, our convergence theorem generalizes and unifies the results of the mentioned literature.

Moreover, if the measure graphs converge to a limit we can interpret the limit of the Zeta functions as the Zeta function of the limit measure graph. In this way, we obtain a continuity statement for the Zeta function.

We start with the following elementary but general observation on the convergence of Zeta functions.

Proposition 5.1. Let $\left(\left(G_{n}, M_{n}\right)\right)$ be a sequence of measure graphs with averaging functions $\left(\mathfrak{u}_{n}\right)$ and vertex sets $\left(V_{n}\right)$ and uniform vertex degree bound $D \in \mathbb{N}$. Assume that the sequence $\int_{V_{n}} \mathfrak{u}_{n}(x) d M_{n}(x)$ is bounded and the limit

$$
F_{j}:=\lim _{n \rightarrow \infty} \int_{V_{n}} N_{j}^{G_{n}}(x) \mathfrak{u}_{n}(x) \mathrm{d} M_{n}(x)
$$

exists for any $j \in \mathbb{N}$. Then

$$
Z_{\left(G_{n}, M_{n}\right)} \rightarrow Z,
$$

uniformly on compact subsets of $B_{(D-1)^{-1}}:=\left\{u \in \mathbb{C}:|u|<(D-1)^{-1}\right\}$, where $Z$ is given by

$$
Z(u)=\exp \left(\sum_{j \geq 1} \frac{F_{j}}{j} u^{j}\right) .
$$


If $(G, M)$ is a measure graph with vertex set $V$ and averaging function $\mathfrak{u}$ which satisfies

$$
F_{j}=\int_{V} N_{j}^{G}(x) \mathfrak{u}(x) \mathrm{d} M(x)
$$

for any $j \in \mathbb{N}$, then $Z=Z_{(G, M)}$.

Proof. By assumption we have convergence of the coefficients

$$
\bar{N}_{j}^{(n)}:=\int_{V_{n}} N_{j}^{G_{n}}(x) \mathfrak{u}_{n}(x) \mathrm{d} M_{n}(x) \rightarrow F_{j} .
$$

Since $\left(M_{n}\left(V_{n}\right)\right)$ is bounded by some constant $C \geq 0$ and $N_{j}$ satisfies $\left|N_{j}(x)\right| \leq D(D-1)^{j-1}$ (cf. proof of Proposition 2.5) we obtain $\bar{N}_{j}^{(n)} \leq C D(D-1)^{j-1}$. This easily gives the desired convergence.

Remark 5.2. Note that boundedness of the sequence $\int_{V_{n}} \mathfrak{u}_{n}(x) d M_{n}(x)$ follows e.g. from boundedness of the sequence $\left(M_{n}\left(V_{n}\right)\right)$.

The previous proposition shows that in order to establish convergence of Zeta functions it suffices to verify pointwise convergence of their coefficients. The following concept of statistical convergence of local patterns will ensure that. Recall that for $\alpha \in \mathcal{A}^{D}$ and $\widetilde{V} \subseteq V$ the set $\widetilde{V}_{\alpha}$ denotes the collection of all vertices in $\widetilde{V}$ whose $\rho(\alpha)$-ball is isomorphic to $\alpha$.

Definition 5.3 (Weakly convergent measure graph sequences). Let $\left(\left(G_{n}, M_{n}\right)\right)$ be a sequence of measure graphs with uniform vertex degree bound $D \in \mathbb{N}$. Let $\left(V_{n}\right)$ be the corresponding vertex sets and let $\left(\mathfrak{u}_{n}\right)$ be the corresponding averaging functions. We call $\left(\left(G_{n}, M_{n}\right)\right)$ weakly convergent if for each $\alpha \in \mathcal{A}^{D}$, the frequency of $\alpha$

$$
p(\alpha):=\lim _{n \rightarrow \infty} \int_{V_{n, \alpha}} \mathfrak{u}_{n}(x) \mathrm{d} M_{n}(x),
$$

exists. In this case, a measure graph $(G, M)$ with vertex set $V$ and averaging function $\mathfrak{u}$ is called a weak limit of $\left(\left(G_{n}, M_{n}\right)\right)$ if for each $\alpha \in \mathcal{A}^{D}$ we have

$$
p(\alpha)=\int_{V_{\alpha}} \mathfrak{u}(x) \mathrm{d} M(x) .
$$

Remark 5.4. Let $G$ be a finite graph equipped with the normalized counting measure on its vertex set and let $\mathfrak{u}$ the function constantly equal to 1 . Then, for $\alpha \in \mathcal{A}^{D}$ we have

$$
\int_{V_{\alpha}} \mathfrak{u} \mathrm{d} M=\frac{\left|\left\{x \in V: \pi_{\rho(\alpha)}(x)=\alpha\right\}\right|}{|V|}=: p(G, \alpha) .
$$

Thus, when interpreted as measure graphs with normalized measure, a sequence of finite graphs $\left(G_{n}\right)$ converges weakly if and only if for any $\alpha \in \mathcal{A}^{D}$ the sequence $\left(p\left(G_{n}, \alpha\right)\right)$ converges. Sequences of connected graphs which satisfy the latter condition were introduced in [3] and called weakly convergent. Therefore, the notion of weak convergence for measure graphs is a generalization. With this observation it is not hard to come up with examples. For instance, considering regular graphs, one obtains such sequences from sofic approximations of groups. Further details including a precise definition for soficity of groups are given in Section 6. Other examples are discussed at the end of this section. 
The next lemma shows that the coefficients of the Zeta function only depend on local patterns. This is the reason why it is compatible with weak convergence. Recall that for a fixed $r \geq 0$ the set $V^{\text {fin,r } r}$ is the collection of all vertices whose rooted connected component has radius $r$. We have seen in Proposition 1.6 that these sets are measurable and naturally appear in a decomposition of $V$ into local patterns. For $\alpha=[(G, x)] \in \mathcal{A}^{D}$ we define

$$
N_{j}(\alpha):=N_{j}^{G}(x) .
$$

This is independent of the particular choice of the representative of $\alpha$.

Lemma 5.5. Let $(G, M)$ be a measure graph an let $j \in \mathbb{N}$. For each natural $n \geq j / 2+1$ the identity

$$
\bar{N}_{j}:=\sum_{\alpha \in \mathcal{A}_{n}^{D}} N_{j}(\alpha) M(\alpha)+\sum_{r=0}^{n-1} \sum_{\alpha \in \mathcal{A}_{r}^{D}} N_{j}(\alpha) M^{\mathrm{fin}}(\alpha)
$$

holds, where

$$
M(\alpha)=\int_{V_{\alpha}} \mathfrak{u d} M
$$

and

$$
M^{\mathrm{fin}}(\alpha)=\int_{V_{\alpha}^{\mathrm{fin}, \rho(\alpha)}} \mathfrak{u} \mathrm{d} M .
$$

Proof. Proposition 1.6 shows that for each $n \in \mathbb{N}$ the set $V$ can be disjointly written as

$$
V=\bigcup_{\alpha \in \mathcal{A}_{n}^{D}} V_{\alpha} \cup \bigcup_{r=0}^{n-1} \bigcup_{\alpha \in \mathcal{A}_{n}^{D}} V_{\alpha}^{\mathrm{fin}, r} .
$$

A closed path of length $j$ starting in $x$ can at most reach the distance $j / 2+1$ from $x$. Therefore, the value $N_{j}(x)$ only depends on the isomorphism class of the ball of radius $j / 2+1$ around $x$. This implies that for $n \geq j / 2+1$ the function $N_{j}$ is constant on each of the sets appearing in the above decomposition. In particular, for $\alpha \in \mathcal{A}_{n}^{D}$ it equals $N_{j}(\alpha)$ on $V_{\alpha}$ and for $\alpha \in \mathcal{A}_{r}^{D}$ with $0 \leq r \leq n-1$ it equals $N_{j}(\alpha)$ on $V_{\alpha}^{\text {fin, }, r}$. Splitting the integral which appears in the definition of $\bar{N}_{j}$ according to this decomposition yields the statement.

In the previous lemma there appeared a term depending on the measure of local patterns in finite connected components. The following lemma shows that these are preserved under weak convergence.

Lemma 5.6. Let $\left(\left(G_{n}, M_{n}\right)\right)$ be a weakly convergent sequence of measure graphs with vertex sets $\left(V_{n}\right)$ and averaging functions $\left(\mathfrak{u}_{n}\right)$. For each $\alpha \in \mathcal{A}^{D}$ the limit

$$
p^{\mathrm{fin}}(\alpha):=\lim _{n \rightarrow \infty} \int_{V_{n, \alpha}^{\mathrm{fin}, \rho(\alpha)}} \mathfrak{u}_{n}(x) \mathrm{d} M_{n}(x)
$$

exists. If $(G, M)$ with vertex set $V$ and averaging function $\mathfrak{u}$ is a weak limit of $\left(\left(G_{n}, M_{n}\right)\right)$ then

$$
p^{\mathrm{fin}}(\alpha)=\int_{V_{\alpha}^{\mathrm{fin}, \rho(\alpha)}} \mathfrak{u}(x) \mathrm{d} M(x)
$$


Proof. As seen in the proof of Proposition 1.6 for each $r \geq 0$ we have

$$
V^{\mathrm{fin}, r}=\bigcup_{\alpha \in \mathcal{A}_{r}^{D}} V_{\alpha} \backslash\left(\bigcup_{\beta \in \mathcal{A}_{r+1}^{D}} V_{\beta}\right) .
$$

For $\alpha \in \mathcal{A}^{D}$ with $\rho(\alpha)=r$ this implies

$$
V_{\alpha}^{\mathrm{fin}, r}=V_{\alpha} \cap V^{\mathrm{fin}, r}=V_{\alpha} \backslash\left(\bigcup_{\beta \in \mathcal{A}_{r+1}^{D}} V_{\beta}\right) .
$$

Since the sets $V_{\beta}$ are pairwise disjoint for different $\beta \in \mathcal{A}_{r+1}^{D}$ the statement follows from the weak convergence.

Theorem 5.7 (Continuity of the Zeta function). Let $\left(\left(G_{n}, M_{n}\right)\right)$ be a weakly convergent sequence of measure graphs with uniform vertex degree bound $D \in \mathbb{N}$. Then there exists a holomorphic function $Z: B_{(D-1)^{-1}} \rightarrow \mathbb{C}$ such that

$$
Z_{\left(G_{n}, M_{n}\right)} \rightarrow Z,
$$

uniformly on compact subsets of $B_{(D-1)^{-1}}$. The function $Z$ is given by

$$
Z(u)=\exp \left(\sum_{j \geq 1} \frac{F_{j}}{j} u^{j}\right),
$$

with

$$
F_{j}=\sum_{\alpha \in \mathcal{A}_{j}^{D}} N_{j}(\alpha) p(\alpha)+\sum_{r=0}^{j-1} \sum_{\alpha \in \mathcal{A}_{r}^{D}} N_{j}(\alpha) p^{\mathrm{fin}}(\alpha) .
$$

In particular, if $(G, M)$ is a weak limit of $\left(\left(G_{n}, M_{n}\right)\right)$, then $Z=Z_{(G, M)}$.

Proof. Together with the definition of weak convergence and Lemma [5.6. Lemma [5.5] shows that the coefficients of $Z_{\left(G_{n}, M_{n}\right)}$ converge and that their limits are given by the $F_{j}$. If $\alpha$ is the class of a graph with one vertex and no edges for any $n$ we have $V_{n, \alpha}=V_{n}$. Therefore, weak convergence implies the boundedness of the sequence $\left(M_{n}\left(V_{n}\right)\right)$. With these observations the statement follows from Proposition 5.1

Remark 5.8. The proof of the previous theorem uses convergence of the statistics of local patterns and that the Zeta functions only depend on these statistics. Therefore, similar statements hold true for objects associated with the graph which only depend on these quantities as well. One example is the integrated density of states of the adjacency operator or other finite range operators in $\mathcal{N}(\mathcal{G}, G)$ (see Section 4 for a definition of the integrated density of states). More precisely, weak convergence of measure graphs implies weak convergence of the integrated density of states. We refrain from giving details.

We finish this section with two applications of our result on continuity of the Zeta function. 
5.1. Weakly convergent graph sequences. As already discussed in Remark 5.4 weakly convergent graph sequences yield weakly convergent measure graphs. It was shown in 12 that for each weakly convergent sequence of connected finite graphs $\left(G_{n}\right)$ with $\left|V_{n}\right| \rightarrow \infty$ there exists a limit graphing $\mathcal{X}$ such that for any $\alpha \in \mathcal{A}^{D}$ we have

$$
\lim _{n \rightarrow \infty} p\left(G_{n}, \alpha\right)=\mu\left(X_{\alpha}\right)=\int_{V_{\mathcal{X}, \alpha}} \mathfrak{u} \mathrm{d} M_{\mathcal{X}}
$$

where $X_{\alpha}=\left\{x \in X: B_{\rho(\alpha)}^{G_{\mathcal{X}}}((x, x)) \in \alpha\right\}$. This implies that the sequence of finite measure graphs $\left(\left(G_{n}, M_{n}\right)\right)$ with normalized counting measure converge weakly to the measure graph $\left(G_{\mathcal{X}}, M_{\mathcal{X}}\right)$. Recall that the Zeta function of a finite graph $G$ with normalized counting measure is denoted by $Z_{G \text {,norm }}$ and that the Zeta function of a graphing $\mathcal{X}$ is denoted by $Z_{\mathcal{X}}$. From Theorem 5.7 and the previous discussion we immediately obtain the following.

Theorem 5.9. Let $\left(G_{n}\right)$ be a weakly convergent sequence of finite connected graphs with uniform vertex degree bound $D \in \mathbb{N}$. There exists a holomorphic function $Z: B_{(D-1)^{-1}} \rightarrow \mathbb{C}$ such that

$$
Z_{G_{n}, \text { norm }} \rightarrow Z
$$

uniformly on compact subsets of $B_{(D-1)^{-1}}$ and the equality

$$
Z=Z_{\mathcal{X}}
$$

holds for every limit graphing $\mathcal{X}$ of $\left(G_{n}\right)$.

Remark 5.10. The above theorem extends the approximation results in the literature: For amenable, periodic graphs (with a discrete, amenable group acting freely and co-finitely as automorphisms), an approximation theorem for the Ihara Zeta function has been shown in [19]. In [6, 16], the authors show the existence of the Ihara Zeta function for certain infinite regular graphs via a convergence statement along covering sequences $\left(G_{n}\right)$ of finite, regular graphs. One way to see the existence of the limit is to use a theorem of Serre 33] on equidistribution of eigenvalues of Markov operators. The convergence along Følner type subgraphs of amenable, self-similar graphs has been proven in [20]. In all the mentioned papers, the graph sequences under consideration can easily be seen to be weakly convergent. Thus, for every approximating sequence given or constructed in the works [6, 16, 19, 20, Theorem 5.7 shows the convergence towards the Zeta function associated to the limit graphing of the sequence. By comparing coefficients, it is not hard to see that in all those cases, this function coincides with the Ihara Zeta function of the original (infinite) graph. (For the periodic case, we verify this explicitly in the proof of Corollary 6.4.) Thus, the above theorem generalizes and unifies the approximation results in the literature.

5.2. Percolation graphs. Let $G_{\Gamma \text {,perc }}$ be the percolation graph of the group $\Gamma$ with respect to the set of generators $I=\left\{g_{1}, \ldots, g_{l}, g_{1}^{-1}, \ldots, g_{l}^{-1}\right\}$. The set of realizations of the percolation $\Theta=\{0,1\}^{\Gamma}$ is a compact space in the product topology and the corresponding Borel $\sigma$-algebra coincides with the product $\sigma$-algebra. Therefore, we have a notion of weak convergence of measures at hand. In the next theorem we prove that it is compatible with weak convergence of the associated measure graphs. We point out here that this also shows that the notion of convergence of measure graph sequences goes beyond weak convergence of finite graphs towards graphings. 
Theorem 5.11. Let $\mathbb{P}$ and $\mathbb{P}_{n}, n \in \mathbb{N}$, be $\Gamma$-invariant probability measures on $\Theta$. If the $\left(\mathbb{P}_{n}\right)$ converge weakly to $\mathbb{P}$ the sequence $\left(\left(G_{\Gamma, \text { perc }}, M_{\mathbb{P}_{n}}\right)\right)$ converges weakly to $\left(G_{\Gamma, \text { perc }}, M_{\mathbb{P}}\right)$. In particular, $\mathbb{P}_{n} \rightarrow \mathbb{P}$ weakly implies

$$
Z_{\left(G_{\Gamma, \text { perc }}, M_{\mathbb{P}_{n}}\right)} \rightarrow Z_{\left(G_{\Gamma, \text { perc }}, M_{\mathbb{P}}\right)}
$$

uniformly on compact subsets of $B_{(2 l-1)^{-1}}$.

Proof. The vertex degree of the percolation graph is at most $2 l$. We have seen that for all $\Gamma$-invariant measures on $\Theta$ the function $\mathfrak{u}=1_{\{e\} \times \Theta}$ is an averaging function. For $\alpha \in \mathcal{A}^{2 l}$ and $n \geq 1$ this implies

$$
\int_{V_{\alpha}} \mathfrak{u d} M_{\mathbb{P}_{n}}=\int_{\Theta} 1_{\left\{\theta \in \Theta: B_{r}^{G}(e) \in \alpha\right\}} \mathrm{d} \mathbb{P}_{n} .
$$

The set $\left\{\theta \in \Theta: B_{r}^{G_{\theta}}(e) \in \alpha\right\}$ is a finite union of cylinder sets in $\Theta$. Therefore, the indicator function in the above integral is continuous with respect to the product topology. From the weak convergence of the measures we infer

$$
\int_{\Theta} 1_{\left\{\theta \in \Theta: B_{r}^{G_{\theta}}(e) \in \alpha\right\}} \mathrm{d} \mathbb{P}_{n} \rightarrow \int_{\Theta} 1_{\left\{\theta \in \Theta: B_{r}^{G_{\theta}}(e) \in \alpha\right\}} \mathrm{d} \mathbb{P}=\int_{V_{\alpha}} \mathfrak{u} \mathrm{d} M_{\mathbb{P}} .
$$

This shows weak convergence of the measure graphs. The 'in particular' statement follows from Theorem 5.7 .

A particular example for the previous theorem is Bernoulli percolation. For $0 \leq p \leq 1$ we let the measure $\mathbb{P}_{p}$ on $\Theta$ be given by

$$
\mathbb{P}_{p}=\bigotimes_{\gamma \in \Gamma}\left[p \delta_{\{0\}}+(1-p) \delta_{\{1\}}\right]
$$

At each vertex independently it deletes all the emerging edges with probability $p$ and keeps them with probability $1-p$. Clearly, the $\mathbb{P}_{p}$ are $\Gamma$-invariant and weakly continuous in the parameter $p$. We obtain

$$
Z_{\left(G_{\Gamma, \mathrm{per} c}, M_{\mathbb{P}_{p}}\right)} \rightarrow 1, \text { as } p \rightarrow 1,
$$

and

$$
Z_{\left(G_{\Gamma, \mathrm{perc}}, M_{\mathbb{P}_{p}}\right)} \rightarrow Z_{\left(G_{0}, \Gamma\right)}, \text { as } p \rightarrow 0,
$$

uniformly on compact subsets of $B_{(2 l-1)^{-1}}$. Here $Z_{\left(G_{0}, \Gamma\right)}$ is the Zeta function of the Cayley graph when viewed as a periodic graph with respect to $\Gamma$.

\section{ACtions OF SOFiC GROUPS ON GRAPHS}

In this section we will investigate approximation of Zeta functions on graphs which are periodic with respect to the action of a sofic group. The key step of our investigation is the construction of a weakly convergent sequence of finite graphs reflecting the local statistics of the (periodic) graph in Theorem 6.3. This will then be combined with the result on weak convergence from the previous section. The assertion of Theorem 6.3 generalizes the previous results provided in 6, 19] in two ways: it is valid for all sofic groups (i.e. not bound to amenable or residually finite groups), as well as for all proper actions on the graph with finite covolume (rather than only for free actions with finite fundamental domain). As the class of sofic groups is rather large we thus obtain a quite general result.

There are various equivalent definitions for soficity of a group. For our purposes, it will be convenient to work with the concept of almost homomorphisms. To give a precise definition, 
we need some preparation. For $n \in \mathbb{N}$, we denote by $\operatorname{Sym}(n)$ the symmetric group over $\{1, \ldots, n\}$ with unit element $\operatorname{Id}_{n}$. This group is naturally endowed with the normalized Hamming distance $d_{H}$, defined as

$$
d_{H}(\sigma, \tau):=\frac{\#\{a \in\{1, \ldots, n\} \mid \sigma(a) \neq \tau(a)\}}{n}
$$

for $\sigma, \tau \in \operatorname{Sym}(n)$. Note that $d_{H}$ is a metric on $\operatorname{Sym}(n)$, see e.g. 28].

Definition 6.1 (Sofic groups). A group $\Gamma$ with unity $e$ is called sofic if for every finite set $T \subseteq \Gamma$ and for each $\varepsilon>0$, there exist $n \in \mathbb{N}$, as well as a mapping

$$
\sigma: T \rightarrow \operatorname{Sym}(n): s \mapsto \sigma_{s}
$$

such that

(i) if $s, t, s t \in T$, then $d_{H}\left(\sigma_{s} \sigma_{t}, \sigma_{s t}\right)<\varepsilon$,

(ii) if $e \in T$, then $d_{H}\left(\sigma_{e}, \operatorname{Id}_{n}\right)<\varepsilon$,

(iii) if $s, t \in T$ with $s \neq t$, then $d_{H}\left(\sigma_{s}, \sigma_{t}\right) \geq 1-\varepsilon$.

If for $T$ and $\varepsilon$, there is some map $\sigma$ satisfying (i), (ii) and (iii), then we say that $\sigma$ is an almost homomorphism for $(T, \varepsilon)$.

Remark 6.2. Sofic groups have been invented by Gromov, cf. [21]. The name was given by Weiss in [37], where the author defined the notion for finitely generated groups. The class of all sofic groups is large. In fact, it is not known whether all groups satisfy this property. Sofic groups have become a flourishing subject in various fields of mathematics, such as geometric group theory, ergodic theory and symbolic dynamics. A survey can be found in [28].

We are now in position to prove the main theorem of this section. Recall that periodic graphs have been introduced in Definition 1.20 ,

Theorem 6.3. Let $(G, \Gamma)$ be a periodic graph with a sofic group $\Gamma$. Assume further that the vertex degree of $G$ is bounded by $D \in \mathbb{N}$ and that $F$ is a fundamental domain of finite co-volume. Then, there exists a weakly convergent sequence of finite graphs $\left(G_{n}\right)$ such that for all $\alpha \in \mathcal{A}^{D}$,

$$
\lim _{n \rightarrow \infty} p\left(G_{n}, \alpha\right)=\frac{\sum_{f \in F_{\alpha}} 1 /\left|\Gamma_{f}\right|}{\sum_{f \in F} 1 /\left|\Gamma_{f}\right|},
$$

where $F_{\alpha}=\left\{f \in F: B_{\rho(\alpha)}^{G}(f) \in \alpha\right\}$.

Proof. Fix an arbitrary $r \in \mathbb{N}, \delta>0$, and a fundamental domain of vertices $F \subset V$ of finite co-volume. The corresponding covering map is denoted by $\pi: V \rightarrow F$. We construct a finite graph $G_{r}$ such that

$$
\left|p\left(G_{r}, \alpha\right)-\left(\sum_{F_{\alpha}} 1 /\left|\Gamma_{f}\right|\right) /\left(\sum_{F} 1 /\left|\Gamma_{f}\right|\right)\right|<g(\delta)
$$

holds for any $\alpha \in \mathcal{A}_{r}^{D}$, where $g:[0,1) \rightarrow \mathbb{R}_{\geq 0}$ is a function solely dependent on $\delta$ with $\lim _{\delta \rightarrow 0} g(\delta)=0$.

We first find some finite $\widetilde{F} \subseteq F$ such that

$$
\sum_{f \in \widetilde{F}} \frac{1}{\left|\Gamma_{f}\right|} \leq \sum_{f \in F} \frac{1}{\left|\Gamma_{f}\right|} \leq \sum_{f \in \widetilde{F}} \frac{1}{\left|\Gamma_{f}\right|}+\delta .
$$


With this notion at hand, we set

$$
\widetilde{B}_{r}^{G}(\widetilde{F}):=\left\{x \in B_{r}^{G}(\widetilde{F}) \mid \pi(x) \in \widetilde{F}\right\}=B_{r}^{G}(\widetilde{F}) \cap \pi^{-1}(\widetilde{F}),
$$

where $B_{r}^{G}(\widetilde{F})$ denotes the union of $r$-balls in $G$ with centers in $\widetilde{F}$. Since the action of $\Gamma$ has finite stabilizers, for each $x \in V$ there exist finitely many elements $\gamma \in \Gamma$ such that $x=\gamma \pi(x)$ holds. We let

$$
T:=T_{\widetilde{F}}:=\left\{\gamma \in \Gamma \mid x=\gamma \pi(x) \text { for some } x \in B_{r}^{G}(\widetilde{F}) \text { with } \pi(x) \in \widetilde{F}\right\}
$$

denote the collection of the $\gamma$ corresponding to elements in the $r$-Ball around $\widetilde{F}$. Note that due to the finiteness of $\widetilde{F}$, as well as the finiteness of the stabilizers, the set $T$ is finite. Note further that for all $f \in \widetilde{F}$, we have $\Gamma_{f} \subseteq T$. We set

$$
\widetilde{T}:=\left(T \cup T^{-1}\right)^{4}=\left\{f g h l \mid f, g, h, l \in T \cup T^{-1}\right\} .
$$

Note that $T \cup T^{-1} \subseteq \widetilde{T}$ since $e \in T$. We now use the fact that $\Gamma$ is sofic in order to find almosthomomorphisms with desirable properties. Precisely, we choose $N \in \mathbb{N}$ and $\sigma: \widetilde{T} \rightarrow \operatorname{Sym}(N)$ such that there is some set $S \subseteq\{1, \ldots, N\}$ with $|S| \geq(1-\delta /|\widetilde{F}|) N$ and for each $i \in S$, we get

(a) $\sigma_{\gamma \gamma^{\prime}}(i)=\sigma_{\gamma} \sigma_{\gamma^{\prime}}(i)$ for all $\gamma, \gamma^{\prime} \in\left(T \cup T^{-1}\right)^{2}$,

(b) $\sigma_{\gamma \gamma^{\prime} \gamma^{\prime \prime}}(i)=\sigma_{\gamma} \sigma_{\gamma^{\prime}} \sigma_{\gamma^{\prime \prime}}(i)$ for all $\gamma, \gamma^{\prime}, \gamma^{\prime \prime} \in T \cup T^{-1}$,

(c) $\sigma_{\theta_{1}}(i)=\sigma_{\theta_{2}}(i)$ if and only if $\theta_{1}=\theta_{2}$ for all $\theta_{1}, \theta_{2} \in \widetilde{T}$,

(d) $\sigma_{e}(i)=i$.

We remark that the existence of such an $N \in \mathbb{N}$, and such a map $\sigma: \widetilde{T} \rightarrow \operatorname{Sym}(N)$ is a straight forward consequence from the definition of soficity by modifying suitable almost homomorphisms in the sense of Definition 6.1. for some $\varepsilon>0$ chosen small enough in terms of $\delta$ and in terms of the sizes of $\widetilde{T}$ and $\widetilde{F}$. By adjusting parameters again if necessary, we can additionally find some set $\tilde{S} \subseteq S$ with

$$
|\tilde{S}| \geq(1-\delta /|\widetilde{F}|)|S| \geq(1-2 \delta /|\widetilde{F}|) N
$$

such that

(e) $\sigma_{\gamma}(i) \in S$ for all $i \in \tilde{S}$ and each $\gamma \in T \cup T^{-1}$.

We will now use this map $\sigma$ to construct the graph $G_{r}$. To this end, we start with an auxiliary construction. For $f \in \widetilde{F}$ and $i, j \in S$ we say that $i$ and $j$ are $f$-pre-connected, if there exists $\gamma \in \Gamma_{f}$ such that $\sigma_{\gamma}(i)=j$. In this case, we write $i \sim_{f} j$.

Claim 1: $\sim_{f}$ is an equivalence relation on $S$. Moreover, for any $i \in \widetilde{S}$ the corresponding equivalence class $[i]_{f}$ satisfies

$$
\left|[i]_{f}\right|=\left|\left\{j \in S \mid i \sim_{f} j\right\}\right|=\left|\Gamma_{f}\right| .
$$

Proof of the claim. We have $e \in \Gamma_{f}$. Hence it follows from (d) that $i \sim_{f} i$ and so $\sim_{f}$ is reflexive. Next we show its symmetry. If $i \sim_{f} j$, then by definition there exists $\gamma \in \Gamma_{f}$ such that $\sigma_{\gamma}(i)=j$. Since $i, j \in S$, properties (a) and (d) yield $\sigma_{\gamma^{-1}}(j)=i$ proving $j \sim_{f} i$. With the same arguments it follows that $i \sim_{f} j$ and $j \sim_{f} k$ implies $i \sim_{f} k$ and so $\sim_{f}$ is transitive. By definition of $\widetilde{S}$ for every $\gamma \in \Gamma_{f}$ and $i \in \widetilde{S}$ we have $\sigma_{\gamma}(i) \in S$ and therefore $i \sim_{f} \sigma_{\gamma}(i)$. Moreover, if $\sigma_{\gamma}(i)=\sigma_{\gamma^{\prime}}(i)$, then (c) implies $\gamma^{\prime}=\gamma$. This proves the desired equality for $\left|[i]_{f}\right|$ and the claim follows. 
This puts us in the position to define the graph $G_{r}$. We let $S_{f}$ the set of equivalence classes of $\sim_{f}$ and $\widetilde{S}_{f}:=\left\{[i]_{f} \in S_{f} \mid i \in \widetilde{S}\right\}$ the set of equivalence classes which have one representative in $\widetilde{S}$. Since the equivalence relation induces a partition of $S$, the previous claim on the size of $[i]_{f}$ for $i \in \widetilde{S}$ yields

$$
\frac{|\widetilde{S}|}{\left|\Gamma_{f}\right|} \leq\left|\widetilde{S}_{f}\right| \leq\left|S_{f}\right| \leq \frac{|S|}{\left|\Gamma_{f}\right|}+|S \backslash \widetilde{S}| .
$$

We define the vertex set of $G_{r}$ as $V_{r}:=\left\{\left(f,[i]_{f}\right) \mid f \in \widetilde{F},[i]_{f} \in S_{f}\right\}$ and we connect two vertices $\left(f,[i]_{f}\right),\left(g,[j]_{g}\right)$ by an edge if there exist $\gamma \in T \Gamma_{f}$ and $\gamma^{\prime} \in T \Gamma_{g}$ such that $\gamma f \sim \gamma^{\prime} g$ in $G$ and $\sigma_{\gamma}(i)=\sigma_{\gamma^{\prime}}(j)$ holds. This definition is independent of the particular choice of $i, j$. Indeed, if $i^{\prime} \in[i]_{f}$ and $j^{\prime} \in[j]_{g}$, then there exist $\gamma_{i} \in \Gamma_{f}$ and $\gamma_{j} \in \Gamma_{g}$ with $\sigma_{\gamma_{i}}\left(i^{\prime}\right)=i$ and $\sigma_{\gamma_{j}}\left(j^{\prime}\right)=j$. Since stabilizers are subgroups we have $\gamma \gamma_{i} \in T \Gamma_{f}$ and $\gamma^{\prime} \gamma_{j} \in T \Gamma_{g}$. From (a) we infer

$$
\sigma_{\gamma \gamma_{i}}\left(i^{\prime}\right)=\sigma_{\gamma}(i)=\sigma_{\gamma^{\prime}}(j)=\sigma_{\gamma^{\prime} \gamma_{j}}\left(j^{\prime}\right)
$$

This implies independence of chosen representatives.

To show the desired statement, for each $i \in \widetilde{S}$, we introduce the maps

$$
\varphi_{i}: \widetilde{B}_{r}^{G}(\widetilde{F}) \rightarrow V_{r}, x \mapsto\left(\pi(x),\left[\sigma_{\gamma^{-1}}(i)\right]_{\pi(x)}\right),
$$

where $\gamma$ solves $x=\gamma \pi(x)$. Note that since $i \in \widetilde{S}$, we have $\sigma_{\gamma^{-1}}(i) \in S$ so that $\left[\sigma_{\gamma^{-1}}(i)\right]_{\pi(x)}$ exists. Naturally, we have to show that this definition does not depend on the choice of $\gamma$. If $\gamma$ and $\gamma^{\prime}$ are two solutions, we necessarily get $\gamma^{-1} \gamma^{\prime} \in \Gamma_{\pi(x)}$. Since $i \in \widetilde{S}$, property (e) combined with the properties (a) and (d) yields

$$
\sigma_{\gamma^{-1} \gamma^{\prime}} \sigma_{\gamma^{\prime}-1}(i) \stackrel{(\mathrm{a})}{=} \sigma_{\gamma^{-1}} \sigma_{\gamma^{\prime}} \sigma_{\gamma^{\prime-1}}(i) \stackrel{(\mathrm{a})}{=} \sigma_{\gamma^{-1}} \sigma_{e}(i) \stackrel{(\mathrm{d})}{=} \sigma_{\gamma^{-1}}(i)
$$

Hence, $\sigma_{\gamma^{\prime-1}}(i) \sim_{\pi(x)} \sigma_{\gamma^{-1}}(i)$.

We need to prove that the mappings $\varphi_{i}$ preserve the local graph structures.

Claim 2: For all $i \in \widetilde{S}$ the map $\varphi_{i}$ is a graph isomorphism onto its image.

Proof of the claim: Let $i \in \widetilde{S}$. We show that $\varphi_{i}$ is injective. For $x, y$ we choose $\gamma_{x}, \gamma_{y} \in T$ with $x=\gamma_{x} \pi(x)$ and $y=\gamma_{y} \pi(y)$. If $\varphi(x)=\varphi(y)$, then $\pi(x)=\pi(y)$ and

$$
\left[\sigma_{\gamma_{x}^{-1}}(i)\right]_{\pi(x)}=\left[\sigma_{\gamma_{y}^{-1}}(i)\right]_{\pi(y)}=\left[\sigma_{\gamma_{y}^{-1}}(i)\right]_{\pi(x)} .
$$

Hence, there exists $\gamma \in \Gamma_{\pi(x)}$ with $\sigma_{\gamma} \sigma_{\gamma_{x}^{-1}}(i)=\sigma_{\gamma_{y}^{-1}}(i)$. By (a) and (c) we obtain $\gamma \gamma_{x}^{-1}=\gamma_{y}^{-1}$. This implies

$$
y=\gamma_{y} \pi(y)=\gamma_{y} \pi(x)=\gamma_{x} \gamma^{-1} \pi(x)=x
$$

and the injectivity is proven.

Next we prove that $\varphi_{i}$ and its inverse preserve edge relations. Let $x, y \in \widetilde{B}_{r}(\widetilde{F})$ with $\left(\pi(x),\left[\sigma_{\gamma_{x}^{-1}}(i)\right]_{\pi(x)}\right) \sim\left(\pi(y),\left[\sigma_{\gamma_{y}^{-1}}(i)\right]_{\pi(y)}\right)$. This is equivalent to the existence of $\gamma \in T \Gamma_{\pi(x)}$ and $\gamma^{\prime} \in T \Gamma_{\pi(y)}$ with $\gamma \pi(x) \sim \gamma^{\prime} \pi(y)$ in $G$ and $\sigma_{\gamma} \sigma_{\gamma_{x}^{-1}}(i)=\sigma_{\gamma^{\prime}} \sigma_{\gamma_{y}^{-1}}(i)$. According to (a) and (c) this in turn is equivalent to the existence of $\gamma \in T \Gamma_{\pi(x)}$ and $\gamma^{\prime} \in T \Gamma_{\pi(y)}$ with $\gamma \pi(x) \sim \gamma^{\prime} \pi(y)$ in $G$ and $\gamma \gamma_{x}^{-1}=\gamma^{\prime} \gamma_{y}^{-1}$. Since $\gamma \gamma_{x}^{-1} x=\gamma \pi(x), \gamma^{\prime} \gamma_{y}^{-1} y=\gamma^{\prime} \pi(y)$ and $\Gamma$ acts on $G$ by graph isomorphisms, the latter statement is equivalent to $x \sim y$ in $G$ and the isomorphism properties for $\varphi_{i}$ are proven.

For $f \in \widetilde{F}$ and $i \in \widetilde{S}$ we have $\varphi_{i}(f)=\left(f,[i]_{f}\right)$. 
To finish the proof of the theorem fix $\alpha \in \mathcal{A}_{r}^{D}$. Since $\mathcal{A}_{r}^{D}$ is finite, by increasing the set $\widetilde{F}$ if necessary (at the beginning of the proof), we can further assume that for the set

$$
\widetilde{F}_{\alpha}:=\left\{f \in F_{\alpha} \cap \widetilde{F} \mid \pi(x) \in \widetilde{F} \text { for all } x \in B_{r}(f)\right\},
$$

we have $\sum_{f \in F_{\alpha}} 1 /\left|\Gamma_{f}\right| \leq \sum_{f \in \widetilde{F}_{\alpha}} 1 /\left|\Gamma_{f}\right|+\delta$. Combining the fact that the $\varphi_{i}$ are graph isomorphisms, which map $f$ to $\left(f,[i]_{f}\right)$, with the bounds in $(\triangle)$, we have

$$
\sum_{f \in \widetilde{F}_{\alpha}} \frac{|\widetilde{S}|}{\left|\Gamma_{f}\right|} \leq\left|\left\{\left(f,[i]_{f}\right) \in V_{r} \mid B_{\rho(\alpha)}^{G_{r}}\left(\left(f,[i]_{f}\right)\right) \in \alpha\right\}\right| \leq \sum_{f \in \widetilde{F}_{\alpha}} \frac{|S|}{\left|\Gamma_{f}\right|}+|\widetilde{F}| \cdot|S \backslash \widetilde{S}| .
$$

Recall that $|S| \leq N$ and by inequality $(\mathrm{O}$, we have

$$
|\widetilde{S}| \geq(1-\delta /|\widetilde{F}|)|S| \geq(1-2 \delta /|\widetilde{F}|) N .
$$

Consequently,

$$
\begin{aligned}
& \sum_{f \in \widetilde{F}_{\alpha}} \frac{(1-2 \delta) N}{\left|\Gamma_{f}\right|} \leq p\left(G_{r}, \alpha\right)\left|V_{r}\right| \leq \sum_{f \in \widetilde{F}_{\alpha}} \frac{N}{\left|\Gamma_{f}\right|}+\delta \cdot N, \\
& \sum_{f \in \widetilde{F}} \frac{(1-2 \delta) N}{\left|\Gamma_{f}\right|} \leq\left|V_{r}\right| \leq \sum_{f \in \widetilde{F}} \frac{N}{\left|\Gamma_{f}\right|}+\delta \cdot N .
\end{aligned}
$$

These inequalities and the fact that the sums over $\widetilde{F}$ and over $\widetilde{F}_{\alpha}$ are close to the sums over $F$, respectively over $F_{\alpha}$ imply

$$
(1-2 \delta) \frac{\sum_{f \in F_{\alpha}} 1 /\left|\Gamma_{f}\right|-\delta}{\sum_{f \in F} 1 /\left|\Gamma_{f}\right|+\delta} \leq p\left(G_{r}, \alpha\right) \leq \frac{1}{1-2 \delta} \frac{\sum_{f \in F_{\alpha}} 1 /\left|\Gamma_{f}\right|+\delta}{\sum_{f \in F} 1 /\left|\Gamma_{f}\right|-\delta} .
$$

With this at hand, routine calculations show that for small $\delta$ the resulting graph $G_{r}$ satisfies inequality $(\nabla)$ with $g(\delta)=C \delta$ for a large enough constant $C$ depending on $\sum_{f \in F} 1 /\left|\Gamma_{f}\right|$ and $\sum_{f \in F_{\alpha}} 1 /\left|\Gamma_{f}\right|$. This finishes the proof.

The previous theorem can be reformulated in terms of weak convergence of measure graphs. It states that for any periodic graph $(G, \Gamma)$ as above there exists a sequence of finite graphs $\left(G_{n}\right)$ such that the associated (normalized) measure graphs $\left(\left(G_{n}, M_{n}\right)\right)$ weakly converge to the measure graph $\left(G,\left(\sum_{f} 1 /\left|\Gamma_{f}\right|\right)^{-1} M\right)$. With this observation we immediately obtain the following from Theorem 5.7 .

Corollary 6.4. Let $(G, \Gamma)$ be a periodic graph with vertex degree bounded by D. Assume further that the group $\Gamma$ is sofic and that $F$ is a fundamental domain of finite co-volume $\mathfrak{v}:=\sum_{f \in F} 1 /\left|\Gamma_{f}\right|$. Then, there is a weakly convergent sequence of finite graphs $\left(G_{n}\right)$ such that

$$
\lim _{n \rightarrow \infty} Z_{G_{n}, \text { norm }}^{\mathfrak{v}}=Z_{(G, \Gamma)},
$$

uniformly on compact subsets of $B_{(D-1)^{-1}}:=\left\{u \in \mathbb{C}:|u|<(D-1)^{-1}\right\}$.

Remark 6.5. (a) The above corollary is an extension of the approximating theorems of [6] dealing with residually finite groups acting freely on a regular graph and [16] dealing with limits of covering sequences of finite, regular graphs. Further, it is the natural extension of the approximation result in [19] which is concerned with amenable graphs. Additional generality is provided by fact that in the framework of a finite measure, we allow for infinite fundamental 
domains, as well as for proper (but not necessarily free) actions of $\Gamma$ on $V$. The crucial idea in the proof for overcoming the lack of freeness is to identify vertices which are linked by almost homomorphisms $\sigma_{\gamma}$ arising from stabilizer elements $\gamma$ ("pre-connectedness").

(b) For graphs with positive Cheeger constant, finite exhaustions might converge, but in general, the geometry of the resulting limit will be completely different from the one of the original objects. One example, as e.g. considered in the introduction of the paper 2, is a sequence of finite, regular trees which converge towards the graphing consisting almost surely of infinite Canopy trees. Those are by no means infinite regular trees. Thus, the approximation through induced subgraphs fails in general in non-amenable situations. However, sofic approximations can still be found in many situations.

(c) The weakly converging sequence of finite graphs constructed in Theorem6.3 for periodic graphs with a sofic group action can also be used for spectral approximation of suitable selfadjoint operators. In fact, whenever a sequence of finite graphs converges weakly then weak convergence of the normalized empirical spectral distributions of corresponding operators to a limit follows and this limit can be expressed through a trace on the von Neumann algebra associated with the limit graphing (see [13, 14, 30, for detailed explanations). In this way, Theorem 6.3 could be used to recover parts of the results of [32]. (The results of 32 are more general in that they allow for unbounded operators and include some randomness.) For hyperfinite graphs even uniform convergence of the normalized empirical spectral distributions can be shown, cf. [13, 30, 29].

(d) Note that the previous corollary is not a special case of Theorem [5.9. Indeed, we identify the limit Zeta function as the Zeta function of a periodic graph instead of that of a graphing. This is possible due to our concept of convergence of measure graphs. This allows us to directly describe the periodic graph as the limit object instead of giving rise to a graphing with the same local statistics as the original graph.

\section{REFERENCES}

[1] H. Bauer. Measure and integration theory. Translated from the German by R. B. Burckel, de Gruyter Studies in Mathematics 26, Berlin, 2001.

[2] I. Benjamini, R. Lyons, O. Schramm. Unimodular random trees, Ergodic Th. Dyn. Sys. 35 (2015), no. 2.

[3] I. Benjamini, O. Schramm. Recurrence of distributional limits of finite planar graphs, Electron. J. Probab. 6 (2001), no. 26.

[4] G. Chinta, J. Jorgenson, A. Karlsson. Heat kernels on regular graphs and generalized Ihara Zeta function formulas, Monatsh. Math. 178, 171-190.

[5] B. Clair, S. Mokhtari-Sharghi. Zeta functions of discrete groups acting on trees, J. Algebra 237 (2001), 591-620.

[6] B. Clair, S. Mokhtari-Sharghi. Convergence of Zeta functions of graphs, Proc. Amer. Math. Soc. 130 (2002), 1881-1886.

[7] B. Clair. The Ihara Zeta function of the infinite grid, Electron. J. Combin. 21 (2014), Paper 2.16.

[8] A. Connes. Sur la théorie non commutative de l'intégration. In Algèbres d'opérateurs (Sém., Les Planssur-Bex, 1978), pages 19-143. Springer, Berlin, 1979.

[9] A. Connes. Noncommutative geometry. Academic Press, San Diego, 1994.

[10] A. Deitmar. Ihara Zeta functions of infinite weighted graphs, SIAM J. discrete Math. 29 (2015), 2100-2116.

[11] J. Dixmier. Von Neumann algebras. North-Holland Publishing Co., Amsterdam, 1981.

[12] G. Elek. On limits of finite graphs, Combinatorica 27 (2007), 503-507.

[13] G. Elek. $L^{2}$-spectral invariants and convergent sequences of finite graphs, J. Funct. Analysis 254 (2008), 2667-2689.

[14] G. Elek. Weak convergence of finite graphs, integrated density of states and a Cheeger type inequality, J. Combin. Theory Ser. B 98 (2008), 62-68.

[15] B. Fuglede, R. Kadison. Determinant theory in finite factors, Ann. Math. (2) 55 (1952), 520-530. 
[16] R. Grigorchuk, A. Zuk. The Ihara Zeta function of infinite graphs, the KNS spectral measure and integrable maps, in: Random walks and geometry, V. Kaimanovich (Ed), (2004), 141-180.

[17] D. Guido, T. Isola. Zeta functions for infinite graphs and functional equations, in: Fractal Geometry and Dynamical Systems in Pure and Applied Mathematics II: Fractals in Applied Mathematics, Carfi, Lapidus, Pearse, and van Frankenhuijsen (Ed), (2013), 123-146

[18] D. Guido, T. Isola, M. L. Lapidus. Ihara's Zeta function for periodic graphs and its approximation in the amenable case, J. Funct. Anal. 255 (2008), 1339-1361.

[19] D. Guido, T. Isola, M. L. Lapidus. Ihara's Zeta function for periodic graphs and its approximation in the amenable case, J. Funct. Anal. 255 (2008), 1339-1361.

[20] D. Guido, T. Isola, M. L. Lapidus. A trace on fractal graphs and the Ihara Zeta function, Trans. Amer. Math. Soc. 361 (2009), 3041-3070.

[21] M. Gromov. Topological invariants of dynmamical systems and spaces of holomorphic maps I, Math. Phys. Ana. Geom. 2 (1999), 323-415.

[22] D. Lenz, F. Pogorzelski, M. Schmidt. Topological measure graphs and inverse semigroups, in preparation.

[23] D. H. Lenz, N. Peyerimhoff, I. Veselić. Groupoids, von Neumann Algebras and the Integrated Density of States, Math. Phys. Anal. Geom., 10 (2007), 1-41.

[24] H. Li, A. Thom. Entropy, Determinants, and $L^{2}$-Torsion, J. Amer. Math. Soc. 27(1) (2014), $239-292$.

[25] L. Lovász. Large Networks and Graph Limits, Amer. Math. Soc., Rhode Island, 2012.

[26] W. Lück. Approximating $L^{2}$-invariants by their finite-dimensional analogues, Geom. Funct. Anal. 4(4) (1994), 455-481.

[27] A. L. T. Paterson : Groupoids, Inverse Semigroups, and their Operator Algebras, Progress in Mathematics, 170, Birkhäuser, Boston, (1998)

[28] V. Pestov. Hyperlinear and sofic groups: a brief guide, Bull. Symbolic Logic 14 (2008), 449-480.

[29] F. Pogorzelski. Convergence theorems for graph sequences, Int. J. Algebra Comp. 24 (2014).

[30] F. Pogorzelski. Banach space-valued ergodic theorems and spectral approximation, Dissertationsschrift Friedrich-Schiller-Universität Jena, 2014.

[31] J. Renault. A groupoid approach to $C^{*}$-algebras, Lecture Notes in Math., 793, Springer, Berlin, 1980.

[32] C. Schumacher, and F. Schwarzenberger. Approximation of the integrated density of states on sofic groups, Ann. Henri Poincaré 16 (2015), 1067-1101.

[33] J. P. Serre. Répartition asymptotique des valeurs propres de l'opérateur de Hecke $T_{p}$ J. Amer. Math. Soc. 10 (1997), 75-102.

[34] H. M. Stark, A. A. Terras. Zeta functions of finite graphs and coverings, Adv. Math. 121 (1996), 126-165.

[35] A. Terras. Zeta functions of graphs, Cambrigde studies in advanced mathematics Vol 128, Cambridge University Press, Cambridge, 2011.

[36] J. Weidmann. Linear Operators in Hilbert Spaces, Grad. Texts in Math. 68, Springer, New York, 1980.

[37] B. Weiss. Sofic groups and dynamical systems in: Ergodic theory and harmonic analysis (Mumbai, 1999). Sankhya, A62(3):350-359, 2000.

Mathematisches Institut, Friedrich Schiller Universität Jena, 07743 Jena, Germany

E-mail address: daniel.lenz@uni-jena.de

Mathematisches Institut, Universität LeipZig, 04109 LeipZig, Germany

E-mail address: felix.pogorzelski@math.uni-leipzig.de

Mathematisches Institut, Friedrich Schiller Universität Jena, 07743 Jena, Germany

E-mail address: schmidt.marcel@uni-jena.de 